\title{
ACUTE EFFECTS OF THE TRAINING MASK ON SHORT-TERM RECOVERY DURING ROWING INTERVALS
}

by

Hayden W. Hess

\author{
A thesis \\ submitted in partial fulfillment \\ of the requirements for the degree of \\ Master of Science in Kinesiology, Biophysical Studies \\ Boise State University
}

December 2017 
(C) 2017

Hayden W. Hess

ALL RIGHTS RESERVED 


\title{
DEFENSE COMMITTEE AND FINAL READING APPROVALS
}

\author{
of the thesis submitted by
}

Hayden W. Hess

Thesis Title: Acute Effects of the Training Mask on Short-Term Recovery During Rowing Intervals

Date of Final Oral Examination: 16 October 2017

The following individuals read and discussed the thesis submitted by student Hayden W. Hess, and they evaluated his presentation and response to questions during the final oral examination. They found that the student passed the final oral examination.

Shawn R. Simonson, Ed.D. Chair, Supervisory Committee

Scott A. Conger, Ph.D.

Member, Supervisory Committee

Jeffrey M. Anderson

Member, Supervisory Committee

The final reading approval of the thesis was granted by Shawn R. Simonson, Ed.D., Chair of the Supervisory Committee. The thesis was approved by the Graduate College. 


\section{DEDICATION}

To those, like me, who have lost a parent too soon. Use their memories as fire to pursue whatever you dream; to make them proud. 


\section{ACKNOWLEDGEMENTS}

First, I would like to thank my Supervisory Committee Chair and faculty mentor, Dr. Shawn Simonson. You have provided me with great support, advice, and encouragement from the beginning of my program at Boise State University. You inspired my love for teaching and education, as well as for research. I would not have been able to continue my education at the Doctoral level without your mentorship. Second, I would like to thank my supervisory committee members, Dr. Scott Conger and Jeff Anderson, for their willingness to assist me and provide guidance throughout my Thesis during this past year - your insights were key in my completion of my study. Third, I must thank both the Kinesiology and Respiratory Care Departments for financial support. Finally, I would like to thank Dylan Warrington for his willingness to be my research assistant. You were critical in completing my data collection.

I also would like to acknowledge my fellow graduate students, Clare Zamzow and Mark Torres, for your friendship and support during the past two years. In addition, I would like to thank all of the Undergraduate and Graduate students that participated in the study. Without you all, I wouldn't have been able to complete this study. 


\begin{abstract}
The purpose of this study was to examine the acute effects of the Training Mask $2.0(\mathrm{TM})$ on performance $(\mathrm{m})$, blood lactate, heart rate recovery (HRR), stroke volume (SV), cardiac output ( $\dot{\mathrm{Q}})$, heart rate variability (HRV), and breath rate recovery (BRR) when used during short-term recovery. Methods: Seven trained males completed two interval training conditions (TM and Sham TM). Each condition consisted of five, 1-min, max effort rows with 3-min recoveries. The TM (or Sham TM) was worn during each 3min recovery. A repeated measures $2 \times 5$ (condition $\mathrm{x}$ interval) ANOVA was used to determine significant main effects for condition or interval. Post-hoc analysis was conducted using a one-way ANOVA to identify differences in conditions or intervals with the Bonferroni adjustment. Results: There were no differences between TM and Sham TM conditions for performance $(\mathrm{m})(p=0.094)$, blood lactate $(p=0.495), \operatorname{HRR}(p$ $=0.533), \mathrm{SV}(p=0.672), \dot{\mathrm{Q}}(p=0.775)$, or HRV $(p=0.158)$, while BRR was improved in the TM condition $(p=0.008)$. Conclusion: The use of the TM during short-term recovery does not improve rowing performance (m), blood lactate, HRR, SV, Q, or HRV during interval training. Contrary to anecdotal reports, the implications for the TM to enhance short-term recovery are not supported.
\end{abstract}




\section{TABLE OF CONTENTS}

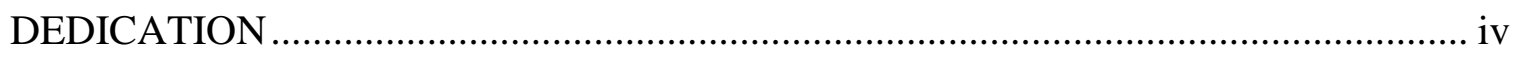

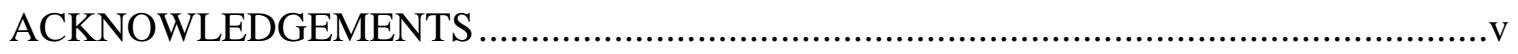

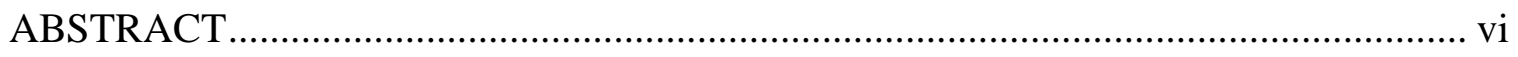

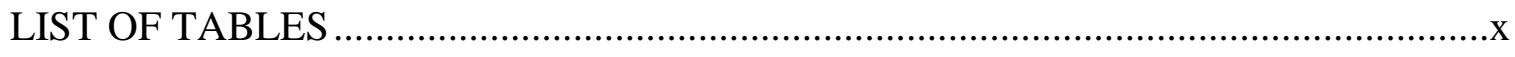

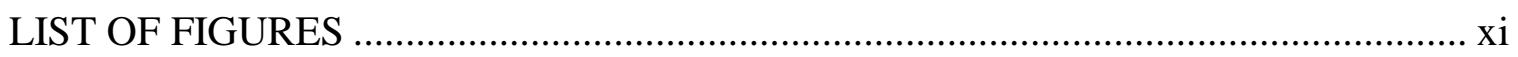

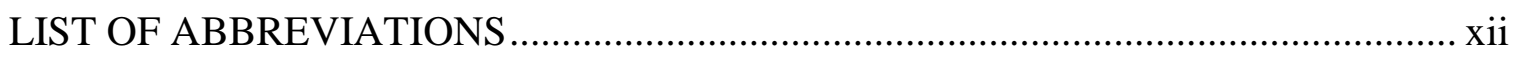

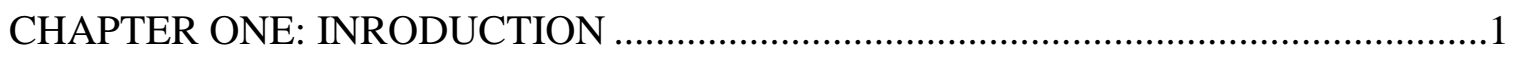

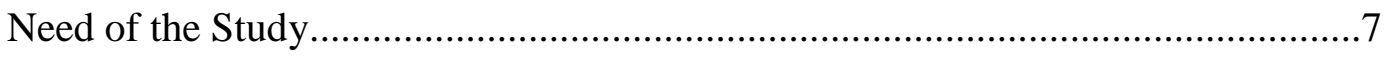

Purpose of the Study ....................................................................................

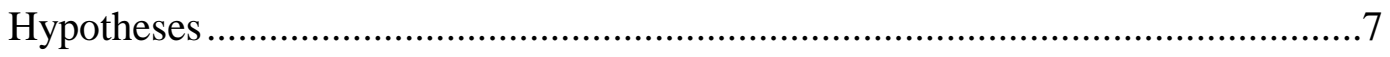

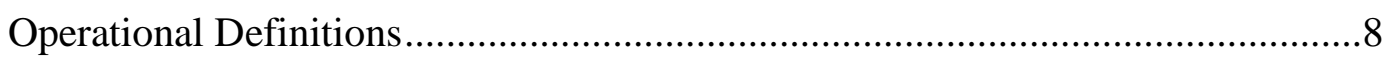

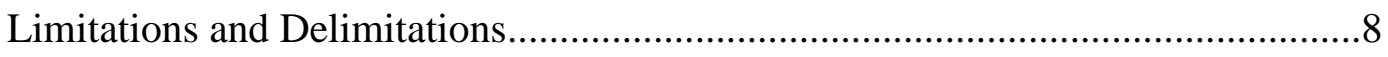

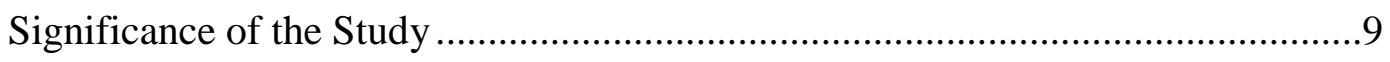

CHAPTER TWO: LITERATURE REVIEW ……………........................................11

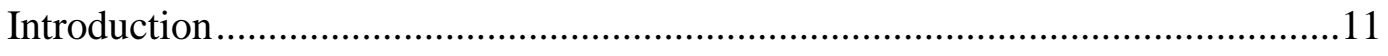

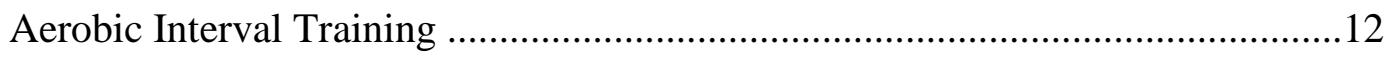

Anaerobic Interval Training ...........................................................................15

Short-Term Recovery During Interval Training ....................................................17

Respiratory Muscle Trainers and the Training Mask ..........................................20 
Potential Mechanisms of the TM on Short-Term Recovery

Shift in Autonomic Nervous System .............................................................22

Venous Return and the "Respiratory Pump" .................................................24

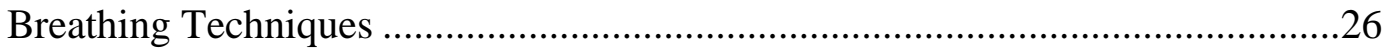

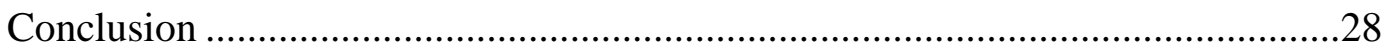

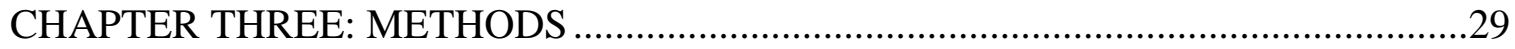

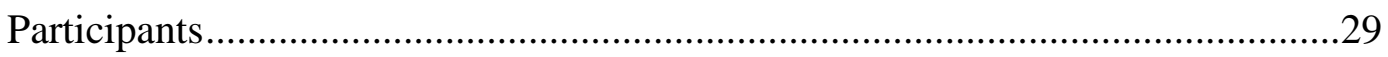

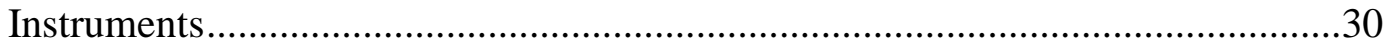

Health History Questionnaire and Modified Physical Activity Readiness

Questionnaire (PAR-Q) (Appendix E) .................................................30

Training Mask 2.0 (Training Mask; Cadillac, MI, USA) (Appendix F)....30

Concept 2 Indoor Rower Model C (Concept2, Inc.; Morrisville, VT,

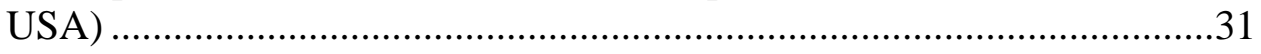

Zephyr Bioharness (Zephyr Technology Ltd.; MD, USA) .......................31

Cheetah Starling-SV (Cheetah Medical, Inc; MA, USA)........................31

Lactate Plus (Nova Biomedical; MA, USA)..........................................32

Visit 1: Briefing, Informed Consent, Health Screening, and Familiarization (Approximately $60 \mathrm{Min}$ )

Visit 2 and 3: Interval Training Protocol Session 1 and 2 (Approximately 60 Min Per Visit)

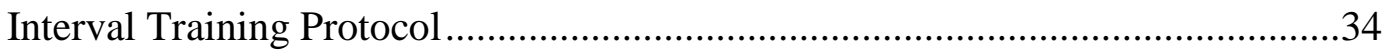

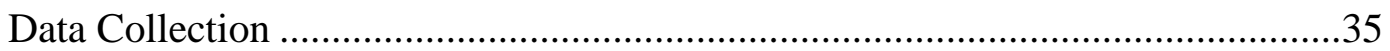

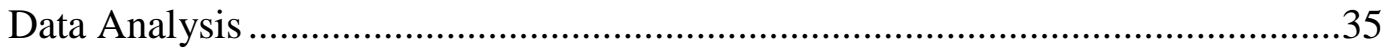

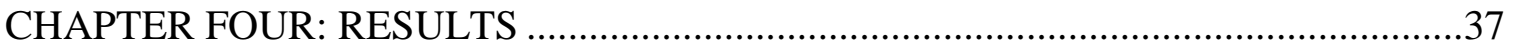

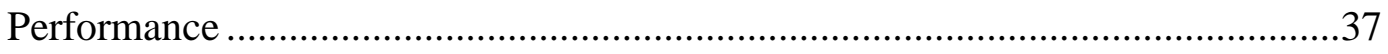




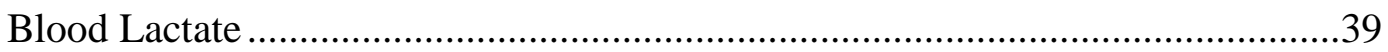

Heart Rate Recovery .............................................................................. 40

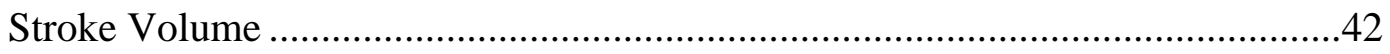

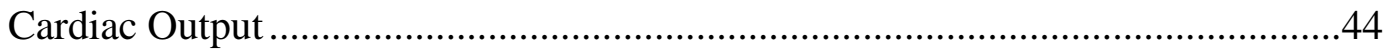

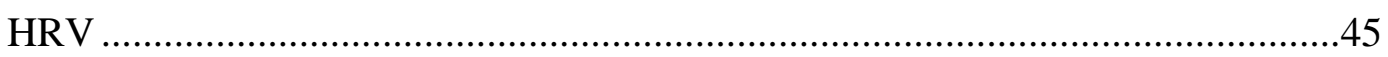

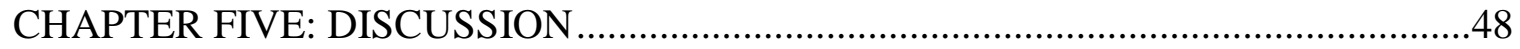

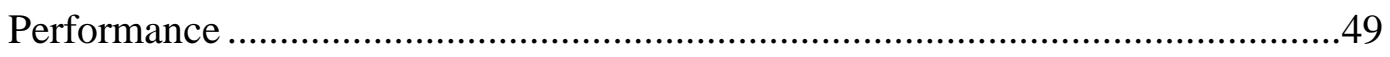

HRR and Potential Mechanisms for Enhanced HRR ........................................52

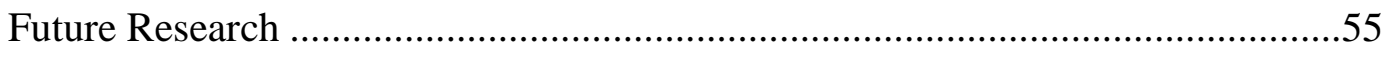

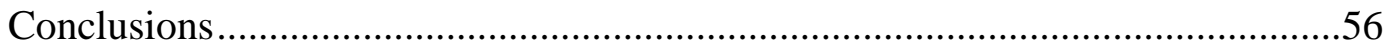

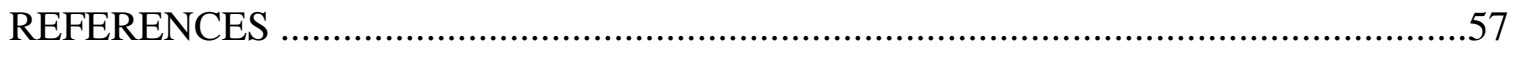

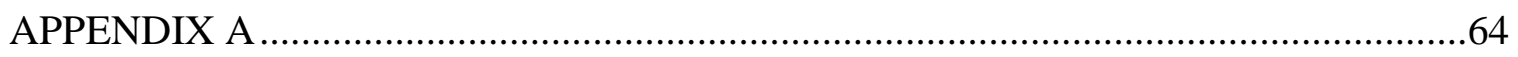

$>\quad$ BENEFITS ......................................................................66

$>\quad$ EXTENT OF CONFIDENTIALITY ........................................66

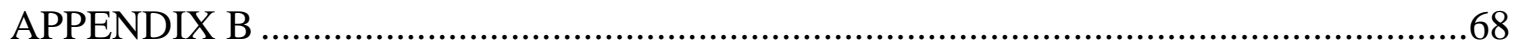

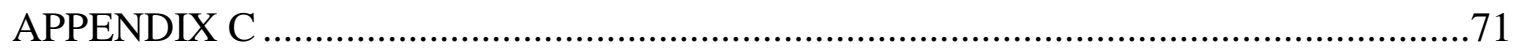

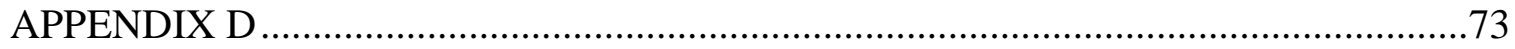

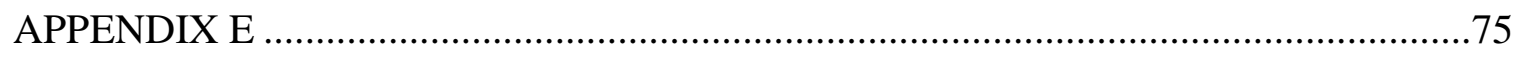

Institutional Review Board Protocol.............................................................. 75 


\section{LIST OF TABLES}

Table 4.1 Participant Characteristics and Training History; $\mathrm{n}=7$ unless otherwise

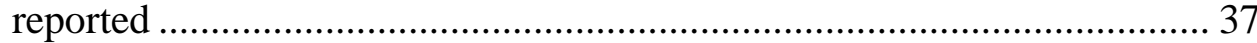

Table 5.1 Hypotheses Following the Results ..................................................... 48 


\section{LIST OF FIGURES}

Figure 1.1 A Right Shift in Velocity-time Relationship Curve from Endurance Training

Figure 4.1 Mean ( \pm SD) Performance $(m)$ for Each Recovery Condition and Interval

Figure 4.2 Mean Performance (m) for TM and Sham TM Conditions. - - -

Responders and — Non-Responders

Figure 4.3 Pre- and Post-Trial Mean $( \pm \mathrm{SD})$ Blood Lactate Concentration $\left(\mathrm{mmol} \cdot \mathrm{L}^{-1}\right)$. *Post-Trial concentrations were significantly greater than pre-trial concentrations in both conditions $(p<0.005)$.

Figure 4.4 Mean $( \pm$ SD) HRR for Each Condition and Interval

Figure 4.5 Mean 3-Min HRR during TM and Sham TM conditions. *Significant difference between responders and non-responders $(p<0.005)$ in TM condition. - - - Responders and - Non-Responders .

Figure 4.6 Mean ( \pm SD) SV during 3-Min Recovery for each Condition and Interval. *Interval one was significantly greater than intervals three, four, and five $(p=0.20,0.21$, and 0.24 , respectively)

Figure 4.7 Mean 3-min Recovery SV for TM and Sham TM Conditions - - -

Responders and — Non-Responders 44

Figure 4.8 Mean ( \pm SD) $\dot{Q}$ during 3-Min Recovery for each Condition and Interval.45

Figure 4.9 Change in Mean $( \pm$ SD) HRV During 3-Min RecoveryBreath Rate Recovery 46

Figure 4.10 Mean ( \pm SD) 3-Min Breath Rate Recovery for Each Condition and Interval. *Significant difference between TM and Sham TM conditions ( $p$ $<0.05)$. 47 
LIST OF ABBREVIATIONS

$\mathrm{VO}_{2}$

$\dot{\mathrm{V}}_{2 \max }$

vنंO 2

ATP

Q

$(\mathrm{a}-\mathrm{v}) \mathrm{O}_{2}$

SV

HR

HRR

HRV

$\min$

sec

HIIT

TM

m

RPE

CT

g

$\mathrm{kg}$

$\mathrm{H}^{+}$
Oxygen Consumption

Maximal Oxygen Consumption or Aerobic Capacity

Velocity at a Specific Oxygen Consumption

Adenosine Triphosphate

Cardiac Output

Arteriovenous Oxygen Difference

Stroke Volume

Heart Rate

Heart Rate Recovery

Heart Rate Variability

Minute(s)

Second(s)

High Intensity Interval Training

Training Mask 2.0

meters

Rating of Perceived Exertion

Traditional Training

Grams

Kilograms

Hydrogen Ion 


$\begin{array}{ll}\text { PPO } & \text { Peak Power Output } \\ \mathrm{mL} & \text { Milliliter(s) } \\ \mathrm{ms} & \text { Millisecond(s) } \\ \text { L } & \text { Liter(s) } \\ \text { PAR-Q } & \text { Physical Activity Readiness Questionnaire } \\ \text { ZB } & \text { Zephyr Bioharness 3.0 } \\ \text { ANOVA } & \text { Analysis of Variance } \\ \text { SD } & \text { Standard Deviation } \\ \text { BRR } & \text { Breath Rate Recovery } \\ \text { RMT } & \text { Respiratory Muscle Training } \\ \text { IMT } & \text { Inspiratory Muscle Training } \\ \text { IRL } & \text { Inspiratory Resistance Loading }\end{array}$




\section{CHAPTER ONE: INRODUCTION}

Endurance can be defined as an individual's ability to maintain a velocity or power output during a single or repeated event (i.e. running, cycling, swimming, rowing, etc.) or competition. ${ }^{1}$ Training methods to enhance endurance are aimed at shifting the velocity-time relationship curve to the right, allowing an individual to perform at an increased velocity or power output for a given distance or time. ${ }^{1,2}$

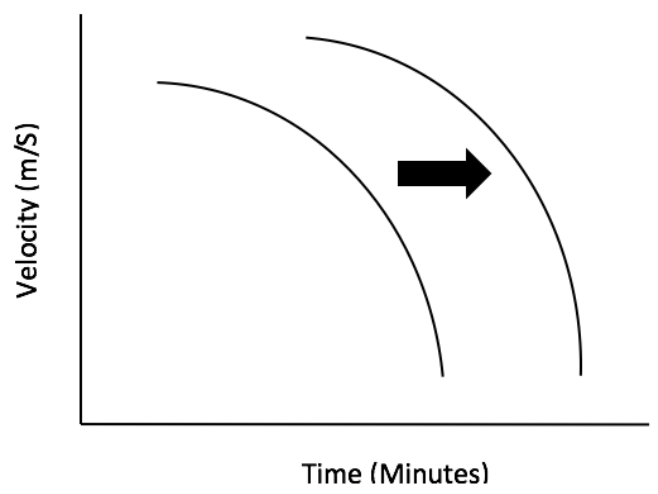

\section{Figure 1.1 A Right Shift in Velocity-time Relationship Curve from Endurance Training}

Both aerobic and anaerobic training methods are utilized by endurance athletes to enhance performance. The determining factor differentiating between the two methodologies is the predominant energy systems utilized during activity. Aerobic training $\left(\leq 100 \%\right.$ aerobic capacity $\left(\dot{\mathrm{VO}}_{2 \max }\right) ;>50 \%$ aerobic energy system contribution; generally $>75 \mathrm{sec}$ ) focuses on enhancing aerobic glycolysis (aerobic synthesis of ATP) and the utilization of carbohydrates and fats as fuel sources. ${ }^{1,3}$ Anaerobic training $(\geq$ $100 \% \dot{\mathrm{VO}_{2 m a x}} ;>50 \%$ anaerobic energy system contribution; generally $<75 \mathrm{sec}$ ) 
enhances the capacity of the phosphagen and anaerobic glycolytic systems to provide energy. ${ }^{3,4}$ Extending the time in which the anaerobic metabolic pathway is contributing to exercise allows the individual to sustain a higher power output or velocity for a longer duration and therefore, leads to enhanced endurance performance. Optimizing endurance training often requires a blend of aerobic and anaerobic intensities. This blend (i.e. interval training) first appeared in peer-reviewed literature in the 1950s, as a successful exercise protocol to enhance endurance performance. ${ }^{2}$

Interval training is defined as repeated short-to-long bouts of either aerobic or anaerobic, high-intensity exercise, generally at or above lactate steady-state velocity (velocity, or intensity, associated with an equilibrium of blood lactate production and removal), with short periods of lower intensity recovery or rest interspersed between the bouts. ${ }^{2,5}$ Billat reviewed the history of interval training and determined implications for the use of aerobic and anaerobic interval training for endurance sports. ${ }^{2}$ Aerobic interval training consists of repeated bouts of work varying from 1 min to long durations (i.e. 8 $20 \mathrm{~min}$ ) at $85-100 \% \dot{\mathrm{VO}}_{2 \max }$. Interval training is anaerobic if the work duration is less than one min, at or above $100 \%$ of an individual's $\dot{\mathrm{V}} \mathrm{O}_{2 \max }$. Anaerobic interval training is to be performed at intensities $\left(\geq 100 \% \dot{\mathrm{V}}_{2 \max }\right)$ and work-to-rest ratios $(\geq 1: 2)$ that maximize overloads of the myocardium and elicit respiratory responses at or above $\dot{\mathrm{V}} \mathrm{O}_{2 \max }{ }^{6}$ For example, Burgomaster et al. employed four to seven "all-out" 30-sec Wingate tests with a 4-min recovery following each work bout. ${ }^{7}$ Appropriately utilized interval training elicits the benefits of increased $\dot{\mathrm{V}}_{2 \max }$ via elevated cardiac output $(\dot{\mathrm{Q}})$ and arteriovenous oxygen difference $\left((\mathrm{a}-\mathrm{v}) \mathrm{O}_{2}\right)$ and are all associated with improved endurance performance. ${ }^{6}$ The increased (a-v) $\mathrm{O}_{2}$ arises from enhanced peripheral oxygen 
extraction at the working tissue level and is a result of increased myoglobin and aerobic enzymes. ${ }^{8}$ The increased $\dot{Q}$ is a result of increased maximal heart rate (HR) and stroke volume $(\mathrm{SV}){ }^{8}$

The autonomic nervous system is an integral part of regulating cardiovascular function. ${ }^{9}$ Parasympathetic activity, or tone, dominates at rest and suppresses HR in healthy populations. HR increases following the commencement of exercise due to parasympathetic secession and sympathetic activation. At the beginning of exercise, initial hemodynamic shifts (i.e. increase in $\dot{Q}$, via increased HR) occur from parasympathetic withdrawal. As exercise intensity increases, sympathetic activation ensues. At maximal or supramaximal intensities, the combination of complete parasympathetic withdrawal and sympathetic activation helps to maintain maximal HR. ${ }^{9}$ The reverse reaction occurs during recovery. At the cessation of exercise, sympathetic activation decreases and parasympathetic increases and heart rate gradually returns to resting levels. However, the process and mechanisms of HR recovery (HRR) at the end of exercise are poorly understood as the role of sympathetic withdrawal and parasympathetic reactivation and the time course associated with the two responses are unclear. ${ }^{9}$ This is of interest because enhanced HRR may play a significant role in sustained performance across multiple intervals - potentially leading to improved training adaptations.

Both short- and long-bout interval training increase $\dot{\mathrm{VO}}_{2 \max }$ and $\mathrm{SV} .^{2,10,11}$ Burgomaster et al also found that just six sessions of repeated Wingate-style interval training (thirty-second maximal effort on the cycle ergometer at a resistance equal to $7.5 \%$ of body weight) enhances muscle oxidative capacity (via increased citrate synthase) 
and doubled time to volitional exhaustion (cycling at $80 \% \dot{\mathrm{VO}}_{2 \text { peak }}, 51 \pm 11$ vs. $26 \pm 9 \mathrm{~min}$ ). ${ }^{7}$ A decrease in submaximal $\mathrm{HR}$ while running five min at $50 \% \dot{\mathrm{VO}}_{2 \max }$ also results from high intensity interval training (HIIT) due to a decrease in sympathetic nervous system drive and increased SV; therefore, allowing the heart more time to fill and enhancing $\dot{Q}$ at a lower HR. ${ }^{4}$ This indicates adaptations of the autonomic nervous system and may provide important implications for examining autonomic function before, during, and after interval training.

HR depends on the interaction of the sympathetic and parasympathetic nervous systems. HR variability (HRV) is a non-invasive method to examine autonomic HR regulation and is determined by measuring the distance between subsequent heart beats by measuring the R-R intervals on an electrocardiogram. ${ }^{9,12-15}$ Decreasing distances between the R-R intervals represents an increase in HR and indicates the withdrawal of the parasympathetic and the activation of the sympathetic nervous system. The reverse, is a marker of parasympathetic reactivation. ${ }^{12}$

HRR can then be used to determine parasympathetic reactivation. Faster HR decay over time is an indication of parasympathetic reactivation following a bout of exercise. ${ }^{13}$ The more highly conditioned an individual is, the faster HRR. ${ }^{13}$ That being said, Buchheit et al found that parasympathetic reactivation may be blunted following a session of repeated sprint intervals. ${ }^{12}$ This may provide insight into performance degradation across repeated intervals. Previous literature has not directly examined parasympathetic reactivation following each work bout of a series of intervals. ${ }^{12,14}$

Few studies have assessed methods to optimize recovery in between interval bouts. Recovery is one of the least understood and researched topics in strength and 
conditioning. Minimal literature exists on methods to enhance short-term, inter-interval recovery. ${ }^{16}$ The few studies that do exist examine the effects of creatine and ginseng supplementation or work and rest durations on short-term recovery. ${ }^{17-20}$ For example, Billat reviewed the literature assessing different work:rest ratios and the subsequent impact of a longer or shorter rest period between exercises. ${ }^{4}$ As would be expected, longer recovery periods resulted in maintenance of performance across each repeated interval and a larger synthesis of phosphocreatine and ATP, through both lactic and alactic anaerobic pathways. ${ }^{4}$ This is important because phosphocreatine availability is the primary limiting factor contributing to fatigue (i.e. decreases in mean or peak power output) during repeated anaerobic intervals. ${ }^{16}$ However, no previous literature, to the author's knowledge, has assessed breathing techniques to enhance short-term recovery during interval training.

Jerath et al assessed the physiology of pranayamic breathing and its capacity to shift the autonomic nervous system towards parasympathetic dominance. ${ }^{21}$ Pranayamic breathing is defined as voluntary breath control and consists of a slow breathing pattern that decreases frequency and increases depth. Slow breathing exercises have been shown to enhance parasympathetic nervous system activation. ${ }^{21}$ Jerath et al concluded that it is evident that ventilation and the parasympathetic nervous system are connected; however, the mechanisms controlling this integration remain unknown. ${ }^{21}$ If slow, rhythmic breathing reactivates the parasympathetic nervous system and decreases sympathetic dominance, studying the effects of this type of breathing used during the recovery portion of the interval may be warranted. 
Slow, rhythmic breathing is a conscious effort that might not be readily achievable following a maximal effort anaerobic interval. A way to 'force' this breathing pattern has been anecdotally examined through the use of the Training Mask 2.0 (TM) (Training Mask; Cadillac, Michigan). Hyperventilation is essentially impossible when the TM is applied to the face. Brian Mackenzie, of Power, Speed, Endurance, has proposed a recovery breathing interval training protocol utilizing the TM during the recovery portion of the training and has reported that well-trained individuals recover faster, as determined by HR, when the mask is applied. ${ }^{22}$ Those who use the TM have reported faster HRR during the rest portion of the interval and better performances (distance covered) across subsequent intervals. The mechanisms that cause this effect of the TM are unknown. A recent study examined the effects of the TM on aerobic capacity, hematological adaptations (hematocrit and hemoglobin), and lung function when applied while completing six weeks of HIIT on a cycle ergometer. ${ }^{23}$ Twenty-four college students were selected for the study. Twelve participants wore the TM during each of the HIIT sessions, while 12 participants did not (control). There was significant improvement in $\dot{\mathrm{V}} \mathrm{O}_{2 \max }$ (13.5\% and 16.5\%) and peak power output (9.9\% and 13.6\%) for the control and TM groups, respectively; however, there were no significant differences between groups. Only the TM group had significant improvement in ventilatory threshold (13.9\%), power output at ventilatory threshold (19.3\%), respiratory compensation threshold (10.2\%), and power output at respiratory compensation threshold (16.4\%). There were no hematological adaptations for either group. The findings reveals that the TM, previously the Elevation Training Mask, does not act as an altitude simulator, but rather a ventilatory resistance device. ${ }^{23}$ This is important to know because the hypotheses for the TM's 
effects on hemodynamics and autonomic nervous system function require the TM to create ventilatory resistance.

\section{Need of the Study}

Currently, a void exists in the literature regarding optimal enhancement of shortterm recovery during the rest portion of HIIT. There is also a lack of literature that examines the effects of ventilatory resistance on hemodynamics during the rest portion of intervals. The TM is a training device designed to imitate various levels of elevation through ventilatory resistance. There is a lack of literature examining the efficacy of the TM's effects (i.e. ventilatory resistance) on short-term recovery and subsequent performance across multiple intervals. Enhancing short-term recovery would allow individuals to sustain a given intensity (i.e. $\% \dot{\mathrm{V}} \mathrm{O}_{2 \max }$ ) or performance (i.e. distance covered) for a longer period of time. Increasing time at high intensities will potentially augment adaptions.

\section{Purpose of the Study}

The purpose of this study was to examine the effects of the TM on HR, HRR, HRV, $\dot{Q}$, and performance across multiple sprint bouts on the rowing ergometer, when the TM is used during the recovery (rest) portion of interval training in well-trained, adult males.

\section{Hypotheses}

Because the TM deepens and slows ventilation, it was hypothesized that due to enhanced autonomic recovery: 1) HRR, HRV, and performance (m rowed) will be greater across each repeated rowing interval when the TM is used during the recovery portion of interval training, 2) the use of the TM will enhance $\dot{Q}$ during the recovery portion of the 
interval, and 3) blood lactate accumulation over the duration of the interval training protocol will be less when the TM is used during the recovery portion of the interval.

\section{Operational Definitions}

- Healthy: No existing medical conditions or injuries, not currently taking medications

- Performance: Distance (m) covered during each 1-min row; Work (W) completed during each 1-min row

- HR Recovery: Difference between HR immediately following a 1-min 'max effort' row and HR following a 3-min recovery ${ }^{9,12}$

- HR Variability: Varying distances between subsequent heart beats, by measuring the time (ms) of R-R intervals on an electrocardiogram and predicting autonomic nervous system function ${ }^{12}$

- Cardiac Output: The total amount of blood being pumped per min by the heart ${ }^{24}$

- Blood Lactate: Lactic acid accumulation in the blood as a result of anaerobic metabolism $^{5}$

- Lactate Steady-State: Represents the highest level of exercise intensity where an equilibrium exists between lactate production and lactate removal ${ }^{5}$

\section{Limitations and Delimitations}

A few limitations were determined prior to conducting the study. Only adult, trained, males were recruited for the study. Only males were recruited because of the potential effects of varying hormonal levels during menstruation in females. ${ }^{25}$ Menstrual status would have to be monitored and the trials conducted at the same time during the menstrual cycle and would increase the cost, complexity, and time to complete the study. 
Therefore, the data resulting from the study may not be generalized to women, elderly or young populations, or untrained individuals. A second possible limitation is a placeboeffect. Within the present protocol, it is impossible to blind the intervention. That being said, there could be psychological effects when the participants are using the TM.

There are a few delimitations that were included in the study. Each participant was required to refrain from exercise for 24 hours prior to each trial. This delimited the potential negative effects of prior exercise on performance during the trials. The participants were required to record their diet and hydration for 24 hours prior to the first trial and submit it to the researcher. The participants were then asked to repeat the diet for 24 hours prior to the second trial in order to delimit the effects of varying energy substrate availability.

\section{Significance of the Study}

The potential significance of the study is to provide empirical data to either support or deny anecdotal reports of success using of the TM as a recovery device when used during the recovery portion of interval training. Another significance of the study would be to potentially support a novel device to enhance short-term recovery. Enhanced short-term recovery will potentially allow individuals to continue to perform HIIT or high intensity interval sport performance at higher levels of intensity, than when short-term recovery fails to be augmented. The present study can provide implications for the use of the TM outside of its intended use. The study has potential to change the scope of the conditioning device. The study also could provide first of its kind literature on the effects of ventilatory resistance on $\mathrm{HRR}, \mathrm{HRV}, \mathrm{SV}, \dot{\mathrm{Q}}$, and performance across multiple 
intervals on the rowing ergometer in trained, adult males. A secondary, but potentially novel aspect of the present study is that it utilizes the rowing ergometer. 


\section{CHAPTER TWO: LITERATURE REVIEW}

\section{Introduction}

An abundance of literature exists attempting to determine optimal ways to enhance endurance capacity, but there is a void in the literature regarding recovery. Bishop et al described recovery as one of the more under-researched and misunderstood parts of the exercise-adaptation cycle. ${ }^{16}$ Strength and conditioning coaches, endurance sport coaches, and athletes often equate more work with more adaptation and ignore the majority of the athlete's time, which is spent in recovery. In order for athletes to optimize training, a sustained level at the target intensity is essential (i.e. $\% \dot{\mathrm{VO}}_{2 \max }$ or pace). The advantage of interval training is the ability to train at higher intensities than can be maintained with continuous training with the result of greater stimulus for adaptation. ${ }^{2}$ In order to accomplish these higher intensities, the recovery in between work bouts, considered short-term recovery, needs to be long enough to sustain a given performance (i.e. velocity, power output, distance). ${ }^{16-18,20}$ In order to sustain high intensities, shortterm recovery must be long enough to allow creatine phosphate repletion $(\sim 170 \mathrm{sec}){ }^{26}$ Research into enhancing short-term recovery has been limited to alterations in duration of recovery (rest) and supplementation (i.e. creatine and ginseng). ${ }^{17-20}$

Recently, Brian Mackenzie, creator of Power, Speed, Endurance, proposed a breathing recovery protocol to enhance short-term recovery using the TM. ${ }^{22}$ Anecdotal reports suggest that using the TM during the rest bouts of interval training results in improved HRR over not using the TM. These anecdotal reports have generated 
mechanistic questions. Does the TM significantly enhance recovery? The TM creates ventilatory resistance and lower-frequency, diaphragmatic or abdominal breathing - what are the effects of this on hemodynamics (i.e. SV and $\dot{Q})$ ? What is the impact on repeated work performance when using the TM during recovery?

This review of the literature is not meant to be exhaustive and comprehensive, but rather attempt to highlight the importance of each possible contributing factor that might affect the success, or failure, of the TM to enhance short-term recovery. The scope of the review of the literature was aerobic and anaerobic interval training, short-term recovery during interval training, and potential mechanisms for enhancing short-term recovery with the TM. The review of the literature is organized by relative importance or effect on the acceptance or rejection of the present hypotheses.

\section{Aerobic Interval Training}

Aerobic interval training was defined by Billat as interval training that stimulates aerobic metabolism more than anaerobic metabolism. ${ }^{2}$ This can be determined by estimating the ratio between the accumulated oxygen deficit and the oxygen consumed during training. Aerobic interval training will elicit a smaller ratio than anaerobic interval training. ${ }^{2}$ More recently, Buchheit and Laursen ${ }^{10}$ reviewed HIIT and renamed aerobic interval training as high-intensity long interval training and defined "long" as work bouts greater than or equal to $60 \mathrm{sec}$ in duration.

Aerobic interval training is utilized to enhance aerobic capacity and endurance sport performance. The work bout of aerobic interval training ranges from 1 to $8 \mathrm{~min}$ in duration with varying periods of the rest interval. Most common rest periods are $30 \mathrm{sec}$ to $5 \mathrm{~min} .{ }^{27}$ The recommended work to rest ratios are $1: 2-4 .^{26}$ The relative intensity of 
aerobic interval training is often between $85-100 \% \dot{\mathrm{VO}}_{2 \text { peak, }}, \dot{\mathrm{V}} \mathrm{O}_{2 \max }$, or peak power output.

Seiler and Sjursen examined the effects of varying work bout durations in aerobic interval training on physiological responses and RPE of 12 well-trained runners ( 9 males, 3 females) completing four different self-paced aerobic interval training protocols on the treadmill over the course of four weeks. ${ }^{27}$ The work interval duration was either 1, 2, 4, or 6 min, with a 1:1 work to rest ratio, meaning the duration of the work interval was equal to the rest interval. The amount of work was fixed at $24 \mathrm{~min}$. The protocols were as follows: $24 \times 1$-min, $12 \times 2$-min, $6 \times 4$-min, or $4 \times 6$-min. It is important to note that the intensity of each interval was self-selected based on RPE. Average running velocity as a percentage of $\dot{\mathrm{V}} \mathrm{O}_{2 \max }\left(\mathrm{v} \dot{\mathrm{V}} \mathrm{O}_{2 \max }\right)$ declined with increasing work interval duration. From $93 \% \mathrm{vV}_{2 \max }$ during the $24 \times 1$-min to $83 \%$ vنंO ${ }_{2 \max }$ during the 4 x 6-min intervals. Peak $\dot{\mathrm{V}}_{2}$ was significantly lower during the $24 \times 1$-min than the other protocols $(82 \pm 5 \%$ $\dot{\mathrm{V}} \mathrm{O}_{2 \max }$ ), while $92-93 \% \dot{\mathrm{V}} \mathrm{O}_{2 \max }$ was reached during the 2-, 4-, and 6-min intervals. $\dot{\mathrm{V}} \mathrm{O}_{2}$ was higher during the recovery interval during the $24 \times 1$-min interval. 1-min of work is not long enough to reach high aerobic pathway contributions and therefore, led to lower $\dot{\mathrm{V}} \mathrm{O}_{2}$. The 1-min work bouts are anaerobic in nature and create a larger oxygen debt and therefore, would lead to higher $\dot{\mathrm{V}} \mathrm{O}_{2}$ during the rest. This is due to the shortest duration of recovery $(1 \mathrm{~min})$. Peak HR was lower during the 1-min intervals than the other protocols. Significant increases in blood lactate only occurred in the 1-min intervals (5.0 $\pm 1.4 \mathrm{vs}$. $\left.3.9 \pm 1.1 \mathrm{mmol} \cdot \mathrm{L}^{-1} ; \mathrm{p}<0.02\right)$. Peak RPE was almost identical across each interval protocol $(16.8 \pm 1.0$ to $17.2 \pm 1.0)$. Key findings of this study include identifying work durations (i.e. 3-5 $\mathrm{min}$ ) that elicit the greatest physiological responses. 
While the majority of aerobic interval training has been focused on running, cycling, and swimming, there are practical applications for aerobic interval training for well-trained rowers. Less literature exists regarding the effects of high-intensity aerobic interval training on rowing, so comparing the improvements of aerobic interval training with using other modalities (i.e. running or cycling) may be difficult to rowing; however, aerobic interval training studies utilizing rowing as the key modality elicit similar physiological adaptations, so there may be crossover that exists.

Ten well-trained rowers ( 5 male, 5 female) completed a baseline 2,000 m rowing time trial, a maximal exercise protocol to determine $\dot{\mathrm{VO}}_{2 \text { peak, a }}$ 4-min all out rowing power test, and a lactate threshold test. ${ }^{11}$ The participants were randomly placed into either a HIIT group or a traditional training (CT) group. The HIIT group completed 8 sets of 2.5 min intervals at $90 \% \mathrm{vVO}_{2 \text { peak, }}$, and the recovery interval was determined by the participants' time to return to 70\% maximal HR. The CT group completed one of two different continuous exercise protocols; either $60 \mathrm{~min}$ at $2-3 \mathrm{mmol} \cdot \mathrm{L}^{-1}$ of blood lactate or $55 \mathrm{~min}$ at 2-3 $\mathrm{mmol} \cdot \mathrm{L}^{-1}$ of blood lactate. Both the HIIT and CT group trained twice per week for 4 weeks. HIIT was associated with significant improvements in 2,000 m time trial performance when compared to the CT group $(1.9 \pm 0.9 \%, \mathrm{p}=0.02)$, but, while both groups increased $\dot{\mathrm{V}} \mathrm{O}_{2 \text { peak, }}$, there were no significant differences in between groups. Driller et $\mathrm{al}^{11}$ did provide the practical implications of the improvement by highlighting the 8 second reduction in a 2,000 m time trial. An $8 \mathrm{sec}$ difference could associate with a 4.5 boat length improvement, compared to the mere 1 boat length improvement from CT. ${ }^{11}$ Selection of work duration is important when programming aerobic interval training because the amount of time spent at or near $\dot{\mathrm{VO}}_{2 \max }$ has been associated with 
higher aerobic capacity adaptations. ${ }^{2,10}$ Fox, Bartels and Billing ${ }^{28}$ stated that repeated bouts of 1 to 8 min of running at $90-100 \% \dot{\mathrm{V}}_{2 \max }$ was optimal for improving $\dot{\mathrm{V}}_{2 \max }$ and performance in endurance athletes. Aerobic interval training often causes acidosis, the primary factor of fatigue during repeated bouts of exercise.${ }^{19}$ Due to this, longer duration intervals (> $8 \mathrm{~min}$ ) do not allow individuals to work at the high percentages of $\dot{\mathrm{V}} \mathrm{O}_{2 \max }$ required for rapid onset acidosis.

Aerobic interval training has been used to enhance aerobic capacity in endurance athletes since before it was first reported in a scientific journal in the 1950 s. $^{2}$ However, recently it has been purported that anaerobic interval training elicits similar aerobic capacity adaptations as aerobic interval training and traditional continuous training. ${ }^{2,10}$

\section{Anaerobic Interval Training}

Anaerobic interval training generally includes any work intervals that are $60 \mathrm{sec}$ or less. ${ }^{4}$ Much anaerobic interval literature focuses on work interval durations around 30 secs, with a work to rest ratio of $1: 3$ or $1: 4 .{ }^{4}$ By definition, the intensity of anaerobic interval training is anaerobic (i.e. $\geq 100 \% \vee \mathrm{V}_{2} \max$ ).

Burgomaster, Hughes, Heigenhauser, Bradwell, and Gibala found that six sessions of sprint interval training can increase muscle oxidative potential and cycle endurance capacity. ${ }^{7}$ Eight recreationally active participants completed a 2 -week sprint interval training intervention and were compared to eight control subjects, who did not complete a training intervention. All participants performed a $\dot{\mathrm{V}} \mathrm{O}_{2 \text { peak }}$ test and a cycle endurance capacity test in which subjects cycled to volitional exhaustion at approximately $80 \% \dot{\mathrm{V}}_{2 \text { peak }}$, before and after the two weeks of sprint interval training. The training entailed six sessions spread over 14 days and consisted of repeated 30 
second maximum sprint efforts on a cycle ergometer. The participants completed 4 - 7 repeated efforts with a 4-min rest in between each work bout. The first session consisted of four repeated efforts and an additional effort was added each training session, except for the sixth session when the subjects only completed four. After two weeks of training, the intervention group improved cycle endurance capacity from $81-169 \%$ compared to baseline ( $51 \pm 11$ min vs. $26 \pm 5 \mathrm{~min} ; p<0.05)$. Resting muscle glycogen increased by $26 \%(p<0.05)$. The data presented showed that with only six training sessions, approximately 15 min of total work over 14 days, are comparable to six training sessions of traditional endurance exercise training ( 2 hours/day at $65 \% \dot{\mathrm{V}}_{2 \text { peak }}$ ) and supports the idea that significantly less training time can elicit similar results to longer durations. A novel result of this study was that it was the first of its kind to show that anaerobic interval training can considerably improve aerobic endurance capacity during a static workload endurance test to exhaustion. ${ }^{7}$ This provides crucial implications for athletes who have a time-limited training schedule. This is not the only study to provide practical application of anaerobic interval training for endurance sports.

Stevens, Olver, and Lemon proposed incorporating anaerobic interval training in combination with traditional endurance training to improve anaerobic capacity and 2,000 m rowing ergometer performance. ${ }^{29}$ Eight subjects were placed into an endurance-based training program without sprint interval training, while eight subjects were placed into an endurance-based training program with sprint interval training replacing 14 of the endurance training sessions with ten sprint interval-training sessions. The decision to eliminate four total sessions was done by the head rowing coach to compensate for increased intensity. Over four weeks, the control group completed 28 rowing ergometer 
training sessions equating to 1,980 min of training time, while the interval group completed 24 rowing ergometer training sessions equating to 1,095 min of training time. Two-thousand m rowing ergometer time trials, $\dot{\mathrm{V}}_{2 \max }$ tests, and 60-sec maximal anaerobic capacity tests were all completed before and after the four weeks of training. It is important to note that a 60 -sec maximal anaerobic capacity test was utilized rather than the traditional "Wingate" style test, because of the nature of rowing and its relatively low-frequency stroke rate, when compared to the pedaling rate of the cycle ergometer. ${ }^{29}$ The sprint interval training sessions consisted of 4-6 repeated 60-sec all-out efforts on the rowing ergometer with 2.5- to 4-min of recovery in between each effort. The results showed that the sprint interval training group significantly improved their 2,000 m rowing ergometer performance when compared to baseline $(414.6 \pm 18.5$ vs. $410.6 \pm 17.5$ sec; $p<0.001)$ and compared to the endurance-based training group $(p=0.03)$. Unlike other sprint interval training studies, $\dot{\mathrm{V}} \mathrm{O}_{2 \max }$ showed no significant changes. ${ }^{4,7,29}$ Peak power output was increased significantly following sprint interval training (566 \pm 82 vs. $623 \pm 60 \mathrm{~W} ; p=0.02)$, as well as average power output $(508 \pm 48 \mathrm{vs.} 530 \pm 52 \mathrm{~W})$. The endurance-based training group showed no significant change in either. ${ }^{29}$

While the findings of the previous two studies detail practical applications of sprint anaerobic interval training for endurance-based sports, there was no discussion regarding recovery strategies to optimize the interval training.

\section{Short-Term Recovery During Interval Training}

Short-term recovery during interval training is an essential determinant of maintained performance. Many questions exist in terms of optimizing short-term recovery. A void exists in the literature examining useful modalities or techniques to 
enhance or optimize recovery. Current literature has focused on the duration of shortterm recovery or the use of supplements to enhance short-term recovery. ${ }^{4,16-18,20}$ Shortterm recovery of power during anaerobic intervals is potentially determined by phosphocreatine synthesis, as phosphocreatine availability is the primary limiting factor contributing to fatigue (i.e. decreases in mean or peak power output) during repeated anaerobic intervals. ${ }^{16,19}$ This explains Cottrell et al's finding that supplementing creatine phosphate increases mean and peak power output across each anaerobic interval. ${ }^{17}$ Thirty trained, male cyclists were placed into one of three groups: 1-, 3-, or 6-min recovery. Each participant completed two trials consisting of eight, 15 -sec all-out bouts on the cycle ergometer at a constant $110 \mathrm{~N}$ of resistance. All thirty participants completed the first trial without supplementation. Six days prior to the second trial, half of each recovery group received either six days of creatine monohydrate supplements in capsules $\left(0.3 \mathrm{~g} \cdot \mathrm{kg}^{-1}\right.$ body weight $)$, while the other half of each recovery group received identical capsules as a placebo, in double-blind fashion. The supplement was taken four times per day, for six days. Following the 6-day supplementation period, each participant completed the second trial of eight, 15-sec all-out bouts on the cycle ergometer. Mean power output was increased significantly for the 1-min recovery, creatine group (694 \pm 25 vs. $739 \pm 26 \mathrm{~W})$, the 3 -min recovery, creatine group $(739 \pm 34$ vs. $791 \pm 31 \mathrm{~W})$, and the 6-min recovery, placebo group ( $807 \pm 16$ vs. $832 \pm 18 \mathrm{~W})(p<0.05)$. Peak power output was significantly increased in the 1-min recovery, creatine group and the 6-min recovery, placebo group ( $848 \pm 26$ vs. $907 \pm 33 \mathrm{~W}$ and $966 \pm 31$ vs. $1021 \pm 34 \mathrm{~W}$, respectively). The key findings were that six days of creatine monohydrate supplementation lead to significant improvements in mean power output across eight, 15- 
sec intervals, when the recovery was 3 min or less. ${ }^{17}$ An interesting finding was that 6min of recovery alone, lead to the highest mean and peak power output, which may indicate that 6 min of recovery may be long enough to replenish muscle phosphocreatine stores without supplementation.

The rest duration may have a crucial impact on short-term recovery. Seiler and Hetlelid examined the effect of three different recovery durations on intensity and RPE during HIIT. ${ }^{20}$ Twelve well-trained distance runners were selected to complete three aerobic interval training sessions consisting of six 4-min bouts of running with either 1-, 2-, or 4-min of recovery between bouts. Running velocity, $\dot{\mathrm{VO}}_{2}$, blood lactate, HR, and RPE data were gathered during the three interval training sessions. Increasing the recovery period from 1- to 2-min was associated with an increase in running velocity, however, not significantly, while no change in running velocity was elicited following a 4-min recovery period. $\dot{\mathrm{VO}}_{2}$, blood lactate, and $\mathrm{HR}$ were not significantly different between the three recovery durations. RPE was highest during the 2-min recovery duration. The key finding of this study was that recovery duration has little effect on running velocity or physiological responses during aerobic interval training. This is important to note because the same is not true for anaerobic interval training. A longer duration (> 2-min) recovery is necessary to avoid acidosis caused from an accumulation of $\mathrm{H}^{+}$following anaerobic metabolism and allow time for phosphocreatine synthesis and clearance of anaerobic metabolites. ${ }^{19}$

Outside of recovery duration and a few supplements, a void exists examining nontraditional modalities or techniques to enhance short-term recovery. A recent study by Pelka, Kolling, Ferrauti, Meyer, Pfeiffer, and Kellmann attempted to determine the best 
psychological relaxation technique to utilize in between two physical tasks. ${ }^{30}$ Twentyseven graduate students completed two sprint interval training sessions consisting of six, 4-sec all-out treadmill sprints with $20 \mathrm{sec}$ of rest in between. In between the two sessions, participants completed $25 \mathrm{~min}$ of one of each of the following recovery strategies: yoga, progressive muscle relaxation, systematic breathing, a power nap, or no intervention. The yoga consisted of a 5 min introductory phase, followed by three rounds of sun salutations, two rounds of triangle A and baddha konaasana, and 5 min of savasana. The progressive muscle relaxation consisted of a 20 min protocol of tensing and relaxing muscle groups. The foundations of the systematic breathing were that exhalation had to be twice as long in duration as inhalation ( $3: 6 \mathrm{sec}$; or, 4:8 sec). The power nap consisted of a $5 \mathrm{~min}$ introduction and a 20 min nap time. The control group was allowed to read. Every participant completed each of the recovery interventions once a week, over a 6-week period, with the first week being a familiarization trial. Systematic breathing lead to the greatest increase in average maximum sprint velocity $(5.17 \pm 0.65 \mathrm{~m} / \mathrm{sec}$ vs. $5.21 \pm 0.61$ $\mathrm{m} / \mathrm{sec}$ ), post-relaxation, but it did not have a significant effect on the other physiological responses or psychological state. ${ }^{30}$ Unfortunately, Pelka et al did not measure autonomic nervous system activity, which may explain the results elicited by the different interventions. ${ }^{30}$ The authors recommended utilizing systematic breathing as a relaxation or recovery modality in between training sessions.

\section{Respiratory Muscle Trainers and the Training Mask}

A recent study examined the effects of wearing the TM on aerobic capacity, lung function, and hematological variables (hematocrit and hemoglobin concentrations). ${ }^{23} \mathrm{~A}$ second, but important purpose of the study, was to determine if the TM acts like an 
altitude simulator. Twenty-five moderately trained college-aged students were recruited to complete a 6-week HIIT protocol using a Monark 828E Ergomedic cycle ergometer. Thirteen participants were placed into the TM group, while the remaining 12 participants were placed into a control group. The TM group wore the TM during all training sessions. Before beginning the 6-week training protocol, all participants completed a maximal cycle ergometer test to determine $\dot{\mathrm{V}}_{2 \max }$, ventilatory threshold, respiratory compensation threshold (the moment when min ventilation increases exponentially with respect to carbon dioxide output), max HR, and peak power output. Pulmonary function was assessed by a forced vital capacity test. Hemoglobin concentration was also determined for each participant. All subjects completed the same training protocol, the difference being the TM. Training sessions were twice a week for $30 \mathrm{~min}$. Each workout included a 5-min warm-up, 20-min HIIT, and a 5-min cool-down. The HIIT protocol consisted of 10 intervals of $30 \mathrm{sec}$ at PPO, followed by a 90 -sec active recovery at $25 \mathrm{~W}$. RPE was recorded following each interval. The power output was increased by $30 \mathrm{~W}$ if the average RPE for two consecutive weeks was $\leq 5$ (hard) or $\leq 7$ (very hard) for the control and TM groups, respectively. The TM's altitude simulation started at $914 \mathrm{~m}$ for week one and increased to $1,829 \mathrm{~m}, 2,743 \mathrm{~m}$, and 3,658 $\mathrm{m}$ for weeks two, three to four, and five to six, respectively. Twelve participants from each group completed the training protocol. Both the control and TM groups significantly increased their $\mathrm{V}_{2 \max }(43.8 \pm 6.4$ vs. $49.5 \pm 7.0 \mathrm{ml} \cdot \mathrm{kg}^{-1} \cdot \mathrm{min}^{-1}$ and $44.8 \pm 6.4$ vs. $52.2 \pm 7.5 \mathrm{ml} \cdot \mathrm{kg}^{-1} \cdot \mathrm{min}^{-1}$, respectively) and peak power output $(+9.9 \%$ and $+13.6 \%$, respectively), but no difference between groups was seen. Only the TM group had significant improvements in ventilatory threshold (+ $14 \%)$, power output at ventilatory threshold $(+19.3 \%)$, respiratory compensation 
threshold $(+10.2 \%)$, and power output at respiratory compensation threshold $(+16.4 \%)$. The TM group had significantly higher respiratory compensation threshold and power output at respiratory compensation threshold compared to the control group $(p<0.05)$. The TM is not an adequate stimulus for hematological changes to occur from hypoxia. The lack of changes in hematological variables and the observation that the TM increases aerobic capacity variables indicates the TM might function like more of a respiratory muscle trainer rather than an altitude simulator. ${ }^{23}$ This is a new finding and has significant implications for the present study, as the hypotheses for the TM's potential for positively effecting HRR and performance rely on its ability to create ventilatory resistance.

\section{Potential Mechanisms of the TM on Short-Term Recovery}

A few potential mechanisms of the TM on short-term recovery can be theorized.

The first theory is that a shift in the autonomic nervous system might occur due to diaphragmatic breathing and the second theory is that modulating breathing techniques and applying ventilator resistance might enhance the "respiratory pump," or the enhanced venous return caused by negative intrathoracic pressure during inspiration. ${ }^{31}$

\section{Shift in Autonomic Nervous System}

There are noninvasive methods to determine autonomic function during recovery following a bout of exercise in humans. The most common methods are to measure parasympathetic reactivation using a time course of HRR and HRV. ${ }^{9,12,14}$ Buchheit et al examined parasympathetic reactivation following repeated sprint exercise in 15 moderately trained individuals. ${ }^{12}$ The participants completed 6 min of repeated $15 \mathrm{~m}$ sprints with $17 \mathrm{sec}$ of recovery in between each bout, a moderate and continuous exercise 
bout $\left(65 \% \dot{\mathrm{V}}_{2 \text { peak }}\right)$, and a high intensity exercise bout consisting of $12 \mathrm{~min}$ of $30 \mathrm{sec}$ runs, followed by $30 \mathrm{sec}$ of recovery. All exercise trials were equal in net energy expenditure. HRR and HRV were measured for 10 min following each of the exercise protocols utilizing the Polar Electro S810. HRR was measured in three ways. First, an absolute HRR was determined for the first $60 \mathrm{sec}$ post exercise by measuring the final exercise HR and the HR after $60 \mathrm{sec}$ of recovery. Second, heart beats during the initial rapid HRR $(10-40 \mathrm{sec})$ were plotted against the time elapsed. Third, HR was plotted on a time-decay curve for 10 min of recovery. HRV was measured by a progressive increase in the R-R interval on a short duration scale (15-60 sec) for $10 \mathrm{~min}$ of recovery. Respiratory rate was not controlled because of the potential disturbance of the natural HRR. The results showed that the repeated sprint exercise protocol elicited a significantly more delayed parasympathetic reactivation during the 10 min recovery. Similarly, Mourot et $\mathrm{al}^{15}$ reported that interval training elicited a slower return of parasympathetic dominance during recovery due to an increased parasympathetic activity withdrawal and/or higher sympathetic involvement following HIIT, compared to continuous, submaximal exercise. Ten moderately trained men were recruited to complete a series of three exercise sessions on the cycle ergometer (incremental exercise test, interval training, and continuous exercise) on three different days, separated by at least one week. The incremental test was used to determine ventilatory threshold and peak and mean power output, $\dot{\mathrm{V}} \mathrm{O}_{2}$, and R-R intervals at ventilatory threshold. During the other two exercise sessions, the participants either completed the interval training (square wave endurance exercise test) or continuous exercise in randomized order. The interval training consisted of nine, 4 min of submaximal exercise at the power output at ventilatory 
threshold, followed by $1 \mathrm{~min}$ at maximal intensity (peak power output). The continuous exercise was performed at power output at ventilatory threshold. Duration of the continuous exercise session was the same as the interval training protocol. Following cessation of each exercise session HRV was measured for the first 20 min post exercise, at 1 hour post-exercise, 24 hours post-exercise, and 48 hours post-exercise. HRV was calculated by R-R interval (ms), total harmonic power and the power of spectral components in low and high frequency. ${ }^{15}$ Short-term exercise effects during the 20 min post-exercise showed there was a decrease in R-R interval, total power, low and high frequency, and the high frequency/total power ratio and an increase in low frequency/total power ratio, low frequency/high frequency ratio for both the interval training and continuous exercise $(p<0.05)$. At one hour post-exercise, $\mathrm{R}-\mathrm{R}$ interval, total power, and low frequency were lower than pre-exercise $(p<0.05)$ for both exercise sessions. High frequency values were lower and low frequency/total power ratio was higher after interval training compared to continuous exercise $(p<0.05)$. Long-term effects of exercise on cardiac autonomic control values were different between the two exercise sessions. Decreased total power and high frequency values between the cessation of exercise and one hour of recovery after interval training, compared to continuous exercise, indicates a slower return of parasympathetic activity during the short-term recovery following HIIT. $^{15}$

\section{Venous Return and the "Respiratory Pump"}

Cyclical variations in intrathoracic and abdominal pressures caused by ventilation play a role in the function of the cardiovascular system. ${ }^{32}$ Increased venous return through negative intrathoracic pressure during ventilation increases stroke volume by 
augmenting atrial filling and therefore increasing cardiac output. ${ }^{24,32,33}$ Miller et al examined the effects of various breathing patterns on venous return and compared the effects to those of skeletal muscle contraction. ${ }^{31}$ Five subjects completed three different breathing patterns with and without calf contractions to determine the differences in femoral venous return. The breathing patterns were normal breathing, ribcage breathing, and diaphragmatic breathing. Ribcage breathing patterns elicited the greatest effect on femoral venous return $(p<0.05)$. Venous return was always positive during ribcage breathing, while diaphragmatic breathing caused a halt in femoral venous return during exhalation. However, during inspiration, femoral venous return was greater in diaphragmatic breathing $(p<0.05)$. That being said, following more than 50 respiratory cycles, there was no difference in femoral venous return between any of the breathing patterns. All statistical analyses were comparisons between breathing patterns and presented as a $p$-value. ${ }^{31}$

Miller et al completed a follow-up study utilizing the same protocol as previously described, but added a respiratory resistance threshold device to augment the inspiratory muscles. ${ }^{34}$ Added inspiratory resistance reduces intrathoracic pressure during inhalation. Subjects were able to increase inspiratory femoral venous return when inspiratory loading was utilized during ribcage breathing, however, despite a consistently increased inspiratory femoral venous return, steady-state femoral venous return (> 50 respiratory cycles) had no significant change during ribcage breathing. Reductions in femoral venous return during expiration lead to a zero net change in total femoral venous return. Femoral venous return while diaphragmatic breathing with inspiratory loading was significantly lower than that of ribcage breathing. The major findings of these two studies were that 
increased negative inspiratory intrathoracic pressure increased the inspiratory femoral venous return during ribcage breathing, but not during diaphragmatic breathing (with and without inspiratory loading). Secondly, enhanced negative intrathoracic pressure during inspiration were incapable of increasing steady-state femoral venous return because of the subsequent offsetting reductions in femoral venous return during expiration. ${ }^{31,34}$

Contrary results were reported by Convertino et al. This study examined the effects of an inspiratory impedance threshold device on hemodynamics in humans. ${ }^{24}$ Inspiratory impedance devices were designed to cause a vacuum-like effect in the chest during inhalation to enhance venous return creating a Frank-Startling effect and increases SV and cardiac output. Twenty subjects completed two trials, one consisted of breathing through an inspiratory impedance threshold device and the second was breathing through a 'placebo' inspiratory device (no resistance). Beat-to-beat recordings of SV were measured noninvasively using a thoracic bioimpedance device. Significant increases in SV (124 \pm 3.0 vs. $137 \pm 3.0 \mathrm{~mL} ; p=0.013)$ and cardiac output $(7.69$ vs. $9.34 \mathrm{~L} / \mathrm{min} ; p=$ 0.001) were reported when the inspiratory impedance threshold device was utilized. A greater vacuum effect within the thorax during each inspiration caused an increase in venous return and a preload or Frank-Starling effect on the heart. The contrasting results of Convertino et al and Miller et al were attributed to the differences in the use of ventilatory resistance. ${ }^{24,31,34}$ Inspiratory resistance alone led to increased venous return, while resistance throughout ventilation led to no change in venous return.

\section{Breathing Techniques}

Few studies exist examining the effects of breathing modulation techniques on the autonomic nervous system. Jerath, Edry, Barnes, and Jerath examined the physiology of 
long pranayamic breathing and its potential to shift the autonomic nervous system. ${ }^{21}$ Pranayama is a voluntary breath control technique often used in yoga practices that consists of a slow inhalation, retention, and a slow exhalation. According to Jerath et al, slow pranayamic breathing can improve or balances the autonomic nervous system. ${ }^{21}$ The authors did not explain potential mechanisms for the positive effects of pranayamic breathing on autonomic nervous system balance. The authors contribute this to an enhanced activation of the parasympathetic nervous system; however, did not employ any methods to test these hypotheses. Jerath et al claims that ventilation and the parasympathetic nervous system are complexly related; however, is not able to provide a mechanism for this purported connection. ${ }^{21}$ Other literature has reported contradicting results.

Patwardhan, Evans, Bruce, Eckberg, and Knapp examined the effects of spontaneous and metronomic breathing on the autonomic nervous system, measured by HRV.$^{35}$ Eight participants performed three separate breathing trials of $10 \mathrm{~min}$. The first was spontaneous breathing (autonomic, involuntary); the second was breathing to a metronome at breathing rates of 15, 18, and 21 breaths per min for 1, 6, and 2 min, respectively; and third was breathing to a metronome at 18 breaths per min for $10 \mathrm{~min}$. The difference between trials two and three were the duration of the metronomic breathing (18 breaths per min). The findings were that when the participants were breathing metronomically, that the voluntary control of the breathing does not alter vagal modulation of HR.

A follow-up study by Patwardhan, Vallurupalli, Evans, Bruce, and Knapp also examined HRV during 5 min of spontaneous and 5 min of controlled breathing. ${ }^{35}$ The 
results showed that voluntary control of breathing caused a decrease in the influence of the parasympathetic nervous system on HR regulation. These findings are on the contrary to the findings of Jerath et al and justifies additional study to clarify the effects of voluntary breath control on HR and HRR.

\section{Conclusion}

Due to a lack of literature on how to modulate hemodynamics during exercise, especially anaerobic interval training, it is warranted to investigate potential modalities for altering ventilation, and to examine its effects on HRR, HRV, and subsequent interval performance. In addition, it is unclear from the literature what the mechanism is for the reported enhanced short-term recovery when using the TM and contradicting evidence for potential mechanics of the untested TM on short-term recovery warrant investigation. 


\section{CHAPTER THREE: METHODS}

\section{Participants}

Seven trained, male rowing ergometer and interval-trained athletes 18 to 45 years old were recruited to participate in this study. The number of participants proposed was based on pilot data, to collect 3-min HRR data, the primary variable, used in a priori power analysis that determined an effect size of 2.094. Performance (m) used in a priori power analysis determined an effect size of .0545 . Based on these effect sizes, the number of participants was determined to be 4 - 5 and 46, respectively. However, the recruitment pool in Boise, ID limits the recruitment of 46 participants that fit the inclusion requirement. The participants were recruited from the Undergraduate and Graduate Kinesiology programs at Boise State University. This research was conducted under approval from the Institutional Review Board at Boise State University, protocol \#103-MED16-008.

The participants were considered trained if they had completed the CrossFit workout "Jackie" (1000 m row, $5045 \mathrm{lb}$ barbell thrusters, and 30 pull-ups) in $\leq 9 \mathrm{~min}$. This time cap was determined by the mean Event 1 (Jackie) times of the 2013 CrossFit Northwest Regional competition (350.9 sec, or 5:50.4 min). ${ }^{36}$ Only male participants were selected for this study because accurate reporting of the menstrual cycle is essential and repeated exercise testing must be conducted during the same menstrual phase. ${ }^{25}$

Participants completed a health history questionnaire and a modified Physical Activity Readiness Questionnaire (PAR-Q) prior to beginning the study. ${ }^{37}$ Exclusion 
criteria were: an orthopedic injury within the past six months, asthma or other lung disease, known heart conditions, or medications that alter exercise capacity. Each participant signed a written Boise State University's Institutional Review Board approved informed consent.

\section{Instruments}

Health History Questionnaire and Modified Physical Activity Readiness Questionnaire (PAR-Q) (Appendix E)

The PAR-Q was developed by the Canadian Society for Exercise Physiology to measure the potential risk of participating in physical activity. It includes questions about heart disease, current medications, musculoskeletal injuries or diseases, and other potential reasons to not participate in physical activity. If the participant answers "no" to all questions he is ready for physical activity. If the participant answers "yes" to any of the questions, he may need to consult with a physician before beginning physical activity. For the sake of the present study, all participants answered "no" to all questions on the PAR-Q.

Training Mask 2.0 (Training Mask; Cadillac, MI, USA) (Appendix F)

The Training Mask 2.0 (TM) is a conditioning device worn over the mouth and nose of the user and is intended to increase respiratory resistance. The TM's purported benefits are: increased diaphragm strength, increased surface area and elasticity of alveoli, increased lung capacity, increased anaerobic threshold, and a decreased workout time by increasing intensity through ventilatory resistance ${ }^{38}$ However, these reported benefits are not supported by peer reviewed literature. The TM, in this study, was used to 
alter ventilatory resistance and $\dot{\mathrm{V}} \mathrm{O}_{2}$. There is currently no validity or reliability evidence for the TM.

Concept 2 Indoor Rower Model C (Concept2, Inc.; Morrisville, VT, USA)

The Concept2 Indoor Rower Model $\mathrm{C}$ is a rowing machine that mimics on-water rowing. The Model C provides estimated data such as distance (m), work (W), calories burned (kcal), and time. The Concept2 Indoor Rower was used in this study to implement the interval training protocol. ${ }^{22}$ Zephyr Bioharness (Zephyr Technology Ltd.; MD, USA)

The Zephyr Bioharness (ZB) is a multivariable physical activity monitoring device, capable of measuring HR, R-R interval, breath frequency, speed, and velocity. The ZB was used to measure HR, R-R interval, and breath frequency. The validity for HR was $r=0.99$ for all data and the validity for breathing frequency was $r=0.94 .{ }^{39}$ Johnstone et $\mathrm{al}^{40}$ also determined that between subject HR and breath frequency reliability was $r=0.70$, while intra- and inter-device data produced a high reliability for HR $(r=>0.89)$. Breath frequency consistently had lower reliability than the other variables.

Cheetah Starling-SV (Cheetah Medical, Inc; MA, USA)

The Cheetah Starling-SV is a non-invasive cardiac output measurement system often found in hospital intensive care units. The Starling-SV utilizes thoracic bioreactance to measure $\dot{Q}$ in liters of blood per min. Bioreactance measures the changes in the electrical conductivity of the thorax due to the shifts in voltage throughout the cardiac cycle. ${ }^{41}$ The Starling-SV utilizes a four-lead placement on the thorax. Compared 
to a Swan-Ganz catheter, the gold standard and invasive measure of cardiac output, the validity for cardiac output was $r=0.84$ and the reliability was $r=0.90 .{ }^{42}$ Lactate Plus (Nova Biomedical; MA, USA)

The Lactate Plus lactate analyzer is a portable, hand-held blood lactate analyzer. It measures blood lactate $\left(\mathrm{mmol} \cdot \mathrm{L}^{-1}\right)$ in $15 \mathrm{sec}$ from a small drop of blood obtained from a finger or earlobe prick. The Lactate Plus has a of validity $(r=0.91)$ compared with a reference blood lactate analyzers (YSI 2300 Stat Analyzer) and had a strong reliability (r $=0.99){ }^{43}$

\section{Procedures}

The study consisted of three visits and took approximately three hours for completion.

$\underline{\text { Visit 1: Briefing, Informed Consent, Health Screening, and Familiarization }}$ $\underline{\text { (Approximately 60 Min) }}$

During the first visit, the researcher met with potential participants in the Human Performance Laboratory at Boise State University. The participants began with completing a Health History Questionnaire and Modified PAR-Q. The researcher provided a briefing including the purposes of the study, the potential risks and benefits of the study, the protocols of the study, and an introduction to the TM. The researcher then answered any questions that the participant had about the study. The participants read and signed the informed consent and were informed that the study was voluntary and that they may withdraw from the study at any point without stating a reason.

The researcher then completed a physical assessment for height $(\mathrm{cm})$ and body mass $(\mathrm{kg})$ of the participant. After the physical assessment, the participants were shown 
how to apply the TM and were given adequate time to become familiar with the use of the TM, the rowing machine, and the interval protocol. The participants were allowed to complete one interval with the TM and one interval with the Sham TM to familiarize themselves with the TM and the interval training protocol.

The participants were then scheduled for the following two visits. They were asked to refrain from exercise for 24 hours prior to each visit and to keep a food log of their diet for 24 hours prior to the next visit.

\section{$\underline{\text { Visit } 2 \text { and 3: Interval Training Protocol Session } 1 \text { and } 2 \text { (Approximately } 60 \text { Min Per }}$} $\underline{\text { Visit) }}$

Upon arrival for the participants' second and third visits, confirmation of 24 hours of no exercise was obtained. The participants reported their 24-hour diet history to the researcher on the second visit. The participants were asked to consume the same 24-hour diet prior to the third visit. The study protocol used was randomly determined to assure that the results are not dependent on the order of the study protocol. On the third visit, the participants completed the other protocol. The participants were fitted with the ZB around the chest, just below the pectoralis line, with the ZB placed on the left anterolateral side of the chest. The Starling-SV electrode pads were applied to four locations on the torso; on the right and left side of the base of the neck, mid-clavicular, and the right and left side just above the iliac crest. The skin was cleaned with rubbing alcohol and cleared of any body hair prior to placing the electrodes on to ensure an unimpeded connection. The lead wires were cleared from obstructing the motion of rowing on the Concept 2 rower. 


\section{Interval Training Protocol}

The participants sat on the Concept 2 indoor rower and placed their feet in the foot straps. The straps were adjusted so that the strap crossed over the top of the metatarsal heads (ball of the foot). The Concept2 indoor rower's damper setting was set at 6.5/10. This is common practice for regular Concept 2 users and the damper setting was fixed so that there was no variance in resistance of the between subjects or visits. The TM was set at a resistance of 3,000 $\mathrm{ft}$., the lowest ventilatory resistance setting for the TM, following the manufactures directions. Prior to beginning the warm-up, the participants' blood lactate $\left(\mathrm{mmol} \cdot \mathrm{L}^{-1}\right)$ was measured via the non-dominant ring finger and analyzed using the Nova Biomedical Lactate Plus lactate analyzer per the manufacturer's instructions. The participants then completed a 5-min, self-paced warm up, without the TM, on the Concept 2 rowing ergometer. The participants then rested for $5 \mathrm{~min}$, or until their HR returned to resting values, before beginning the interval training protocol. At this time, the protocol was briefed for the participant. The interval training protocol consisted of five, 1-min maximal effort rows, interspersed with three min of passive recovery. The work and recovery durations were selected to test the breathing recovery protocol proposed by Brian MacKenzie. ${ }^{22}$ In the treatment condition, the TM was placed on the participant immediately following the 1-min row and worn during the short-term recovery bout, and taken off immediately before beginning the subsequent 1 -min row. ${ }^{22}$ The participant stayed seated on the Concept 2 rowing ergometer and were asked to remain as still as possible during each recovery. All participants unstrapped their feet during the recovery. The participant completed all five intervals, including a 3-min recovery following the final interval. The control condition consisted of the same 
protocol with a Sham TM (without resistance) during the short-term recovery portion of the interval training protocol. One min following the final interval, blood lactate was measured again, but via the earlobe to delimit any potential effects grip on blood lactate concentrations. There are no significant differences between fingertip and earlobe sample site measurements. ${ }^{44}$ The participant then completed a 5-min self-paced cool down.

\section{Data Collection}

HR, HRR, HRV, breath rate, SV, $\dot{Q}$, and performance (m) were collected during the interval training protocol. HR was recorded immediately following each of the 5,1 min rows, and at the end of each 3-min recovery. The difference between the participants' HR following the 1-min row and at the end of the 3-min was recorded. This was the participants' 3-min HRR. The ZB continuously recorded the R-R interval (a measure of HRV) throughout the entire interval training protocol. The difference between the distance of the R-R interval following the 1-min row and the 3-min recovery was recorded for each of the 5 intervals. The ZB recorded breath frequency during the 3-min recovery of each of the intervals. The difference between breath rate following the 1-min row and the end of the 3-min rest was recorded. This was the participants' breath rate recovery. The performance of each 1-min row was also recorded. This was the distance (m) covered in one min.

\section{Data Analysis}

An exploratory data analysis was conducted prior to beginning the statistical analysis to determine if there were any outliers or issues with the data collected. Descriptive statistics (mean \pm SD) for each variable measured were calculated. The hypotheses of the study were that the TM would increase HRR, HRV, Q, SV, breath rate 
recovery, performance $(\mathrm{m})$ during recovery, while decreasing blood lactate concentrations following the complete interval training protocol. The participants completed the interval training protocol with the TM and a Sham TM. Due to the crossover nature of the study protocol, a repeated-measures ANOVA (2X5 within-group; condition X interval) for each of the variables: 3-min HRR, HRV, Q, SV, performance (m), blood lactate, and 3-min breath rate recovery were completed to determine if there were differences between the TM and Sham TM conditions. When appropriate, further post-hoc analysis was completed using a one-way ANOVA to identify differences in conditions or intervals with the Bonferroni adjustment. Significance level was set at $p<$ 0.05. All analyses were completed on SPSS 24.0 (SPSS Inc., Armonk, NY). 


\section{CHAPTER FOUR: RESULTS}

Seven trained, male participants completed the study. One participant dropped out after completing visits one and two and their data was omitted. Participants' physical characteristics and training history are displayed in Table 4.1.

Table 4.1 Participant Characteristics and Training History; $n=7$ unless otherwise reported

\begin{tabular}{|l|l|}
\hline & Mean \pm SD \\
\hline Age $(\mathbf{y})$ & $25.29 \pm 3.15$ \\
\hline Height $(\mathbf{c m})$ & $180.58 \pm 4.44$ \\
\hline Weight $(\mathbf{k g})$ & $84.48 \pm 6.25$ \\
\hline $\begin{array}{l}\text { Years of rowing ergometer and interval training } \\
\text { Participation }(\boldsymbol{n}=\mathbf{4})\end{array}$ & $1.89 \pm 2.51$ \\
\hline Number of Training Sessions Per Week $(\boldsymbol{n}=\mathbf{4})$ & $3.50 \pm 1.29$ \\
\hline Hours Per Training Session $(\boldsymbol{n}=\mathbf{4})$ & $1.13 \pm 0.25$ \\
\hline Jackie Time (sec) & $463.86 \pm 31.02$ \\
\hline 2000m Row Time $(\mathbf{s e c})(\boldsymbol{n}=\mathbf{3})$ & $426.33 \pm 21.50$ \\
\hline
\end{tabular}

\section{Performance}

Descriptive statistics for mean performance (m) are presented in Figure 4.1. Repeated measures ANOVA for performance $(\mathrm{m})$ found a significant main effect for interval in both the TM and Sham TM conditions ( $p=0.003$ and 0.013 , respectively); however, upon post hoc analysis for simple main effects, the data violated Mauchly's Test of Sphericity $(p<0.05)$ due to a small sample size and large variances of individual 
data and there were no significant differences between intervals $(p>0.05)$. There were no significant main effects for either trial condition or trial condition*interval interaction $(p$ $=0.094$ and 0.069 , respectively). Upon further analysis, it was determined that there were four responders and three non-responders of the TM (Figure 4.2). A TM responder was defined as a participant that had increased HRR in the TM condition.

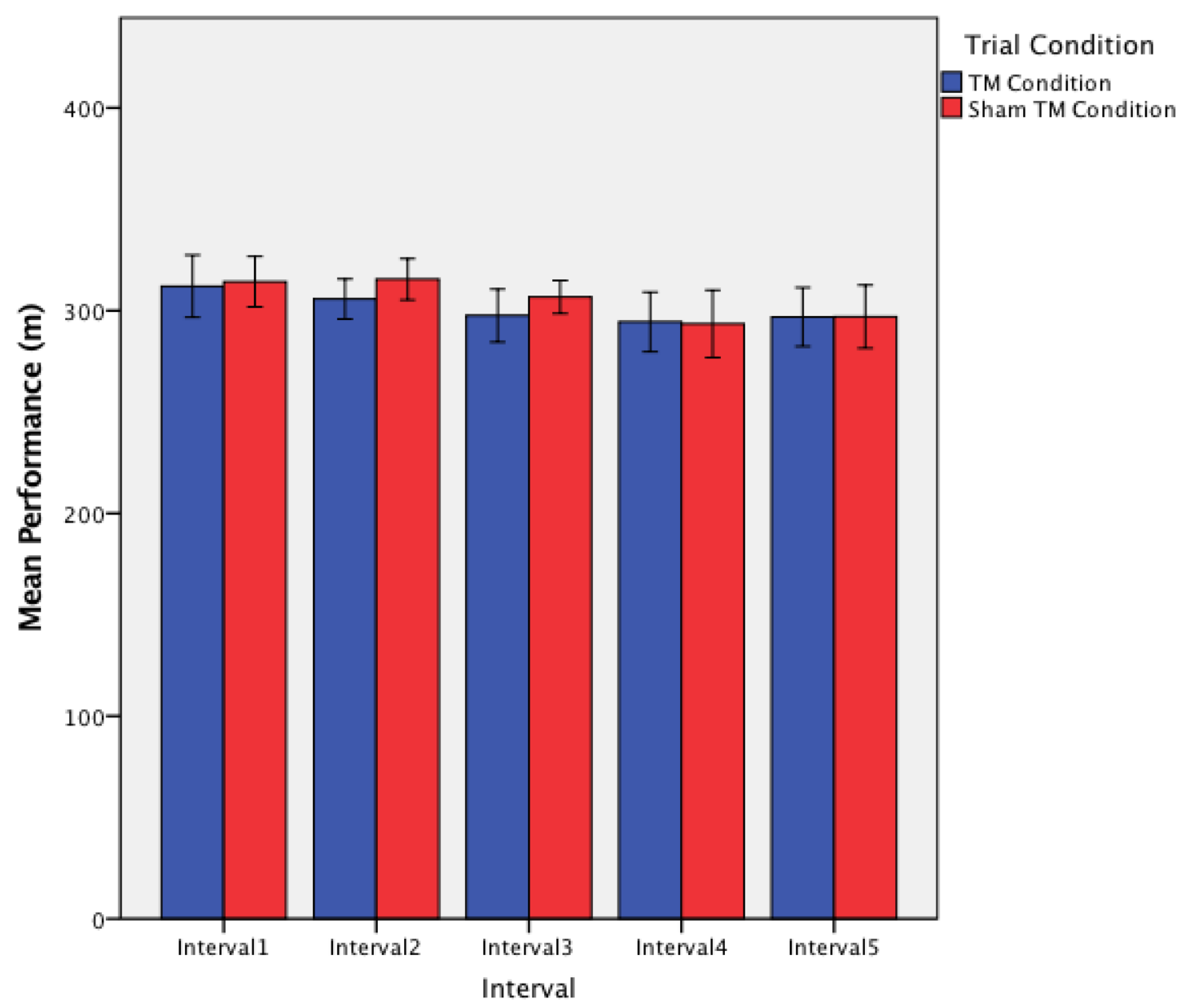

Figure 4.1 Mean ( \pm SD) Performance $(m)$ for Each Recovery Condition and Interval 


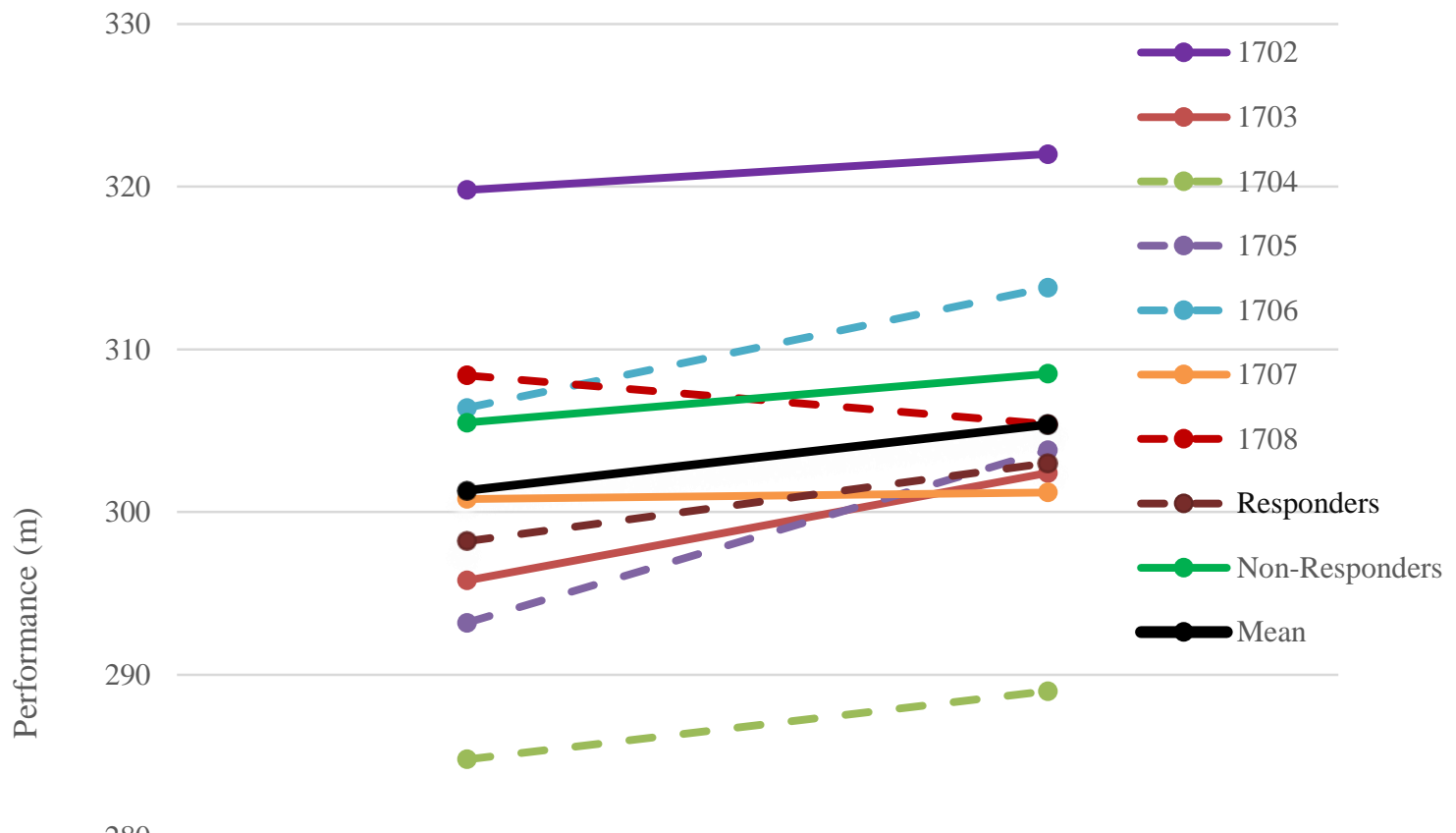

280

270

260

TM

Sham TM

Trial Condition

Figure 4.2 Mean Performance (m) for TM and Sham TM Conditions. - - Responders and — Non-Responders

\section{Blood Lactate}

Blood lactate was measured at two time points in each condition: pre- and posttrial. Descriptive statistics are presented in Figure 4.3. A repeated measures ANOVA found a significant main effect for time (pre- to post-trial) was observed $(p<0.005)$. Post-trial blood lactate concentrations were significantly greater than pre-trial in both conditions $(p<0.005)$. However, no significant main effect for trial condition or trial condition*time ( $p=0.495$ and 0.093 , respectively) was found. 


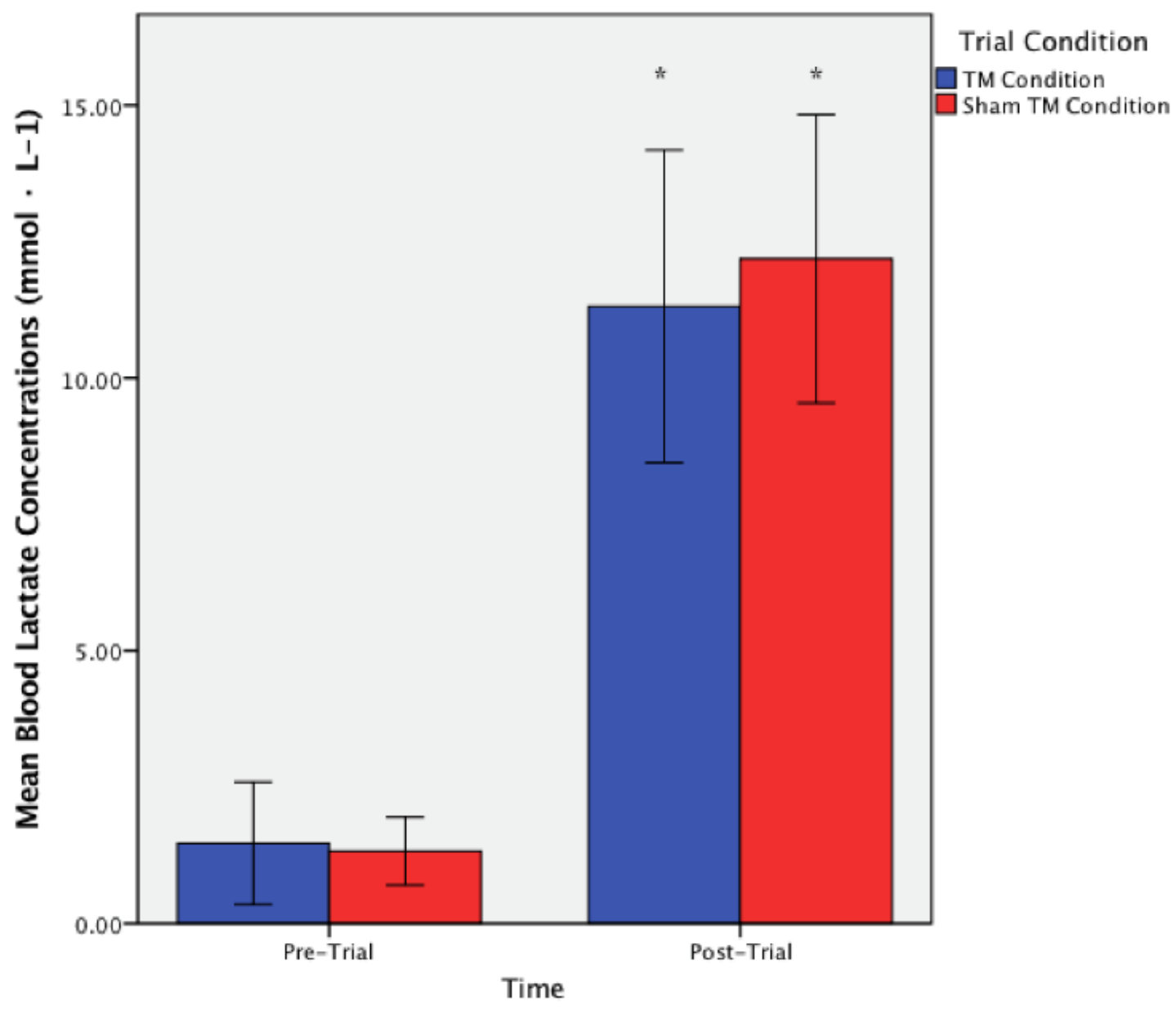

Figure 4.3 Pre- and Post-Trial Mean $( \pm$ SD) Blood Lactate Concentration $\left(\mathrm{mmol} \cdot \mathrm{L}^{-1}\right)$. *Post-Trial concentrations were significantly greater than pre-trial concentrations in both conditions $(p<0.005)$.

\section{Heart Rate Recovery}

HRR was calculated by subtracting the maximum HR following the 1-min row by the final HR following the 3-min recovery. Descriptive statistics are presented in Figure 4.3. Figure 4.4 shows each participant's mean 3 -min HRR for each condition. For HRR a repeated measures ANOVA found no significant main effect for trial condition or trial condition*interval $(p=0.533$ and 0.777$)$. However, a significant main effect for interval $(p=0.028)$. The HRR interval data for both TM and Sham TM conditions violate Mauchly's Test for Sphericity ( $p=0.043$ ); therefore, upon post hoc analysis the TM 
HRR interval data was not significantly different $(p=.184)$, while Sham TM interval data showed a simple main effect of 0.016 ; however, upon analysis of individual interval differences there were no intervals significantly different than each other $(p>0.05)$. Responders had a significantly greater HRR than non-responders in the TM condition ( $p$ $<0.005)$, while there was no difference between responders and non-responders in the Sham TM condition $(p<0.305)$.

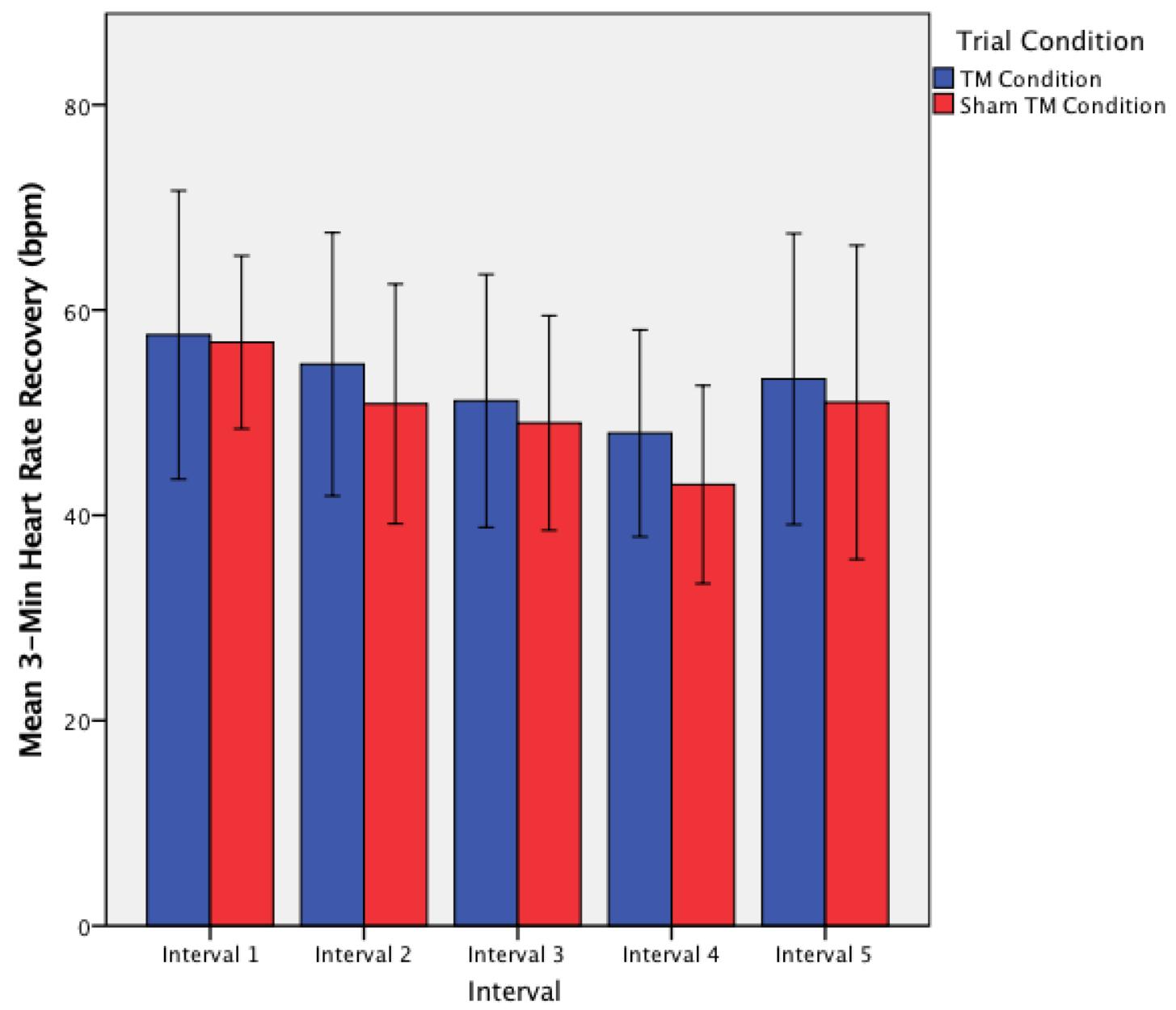

Figure 4.4 Mean $( \pm$ SD) HRR for Each Condition and Interval 


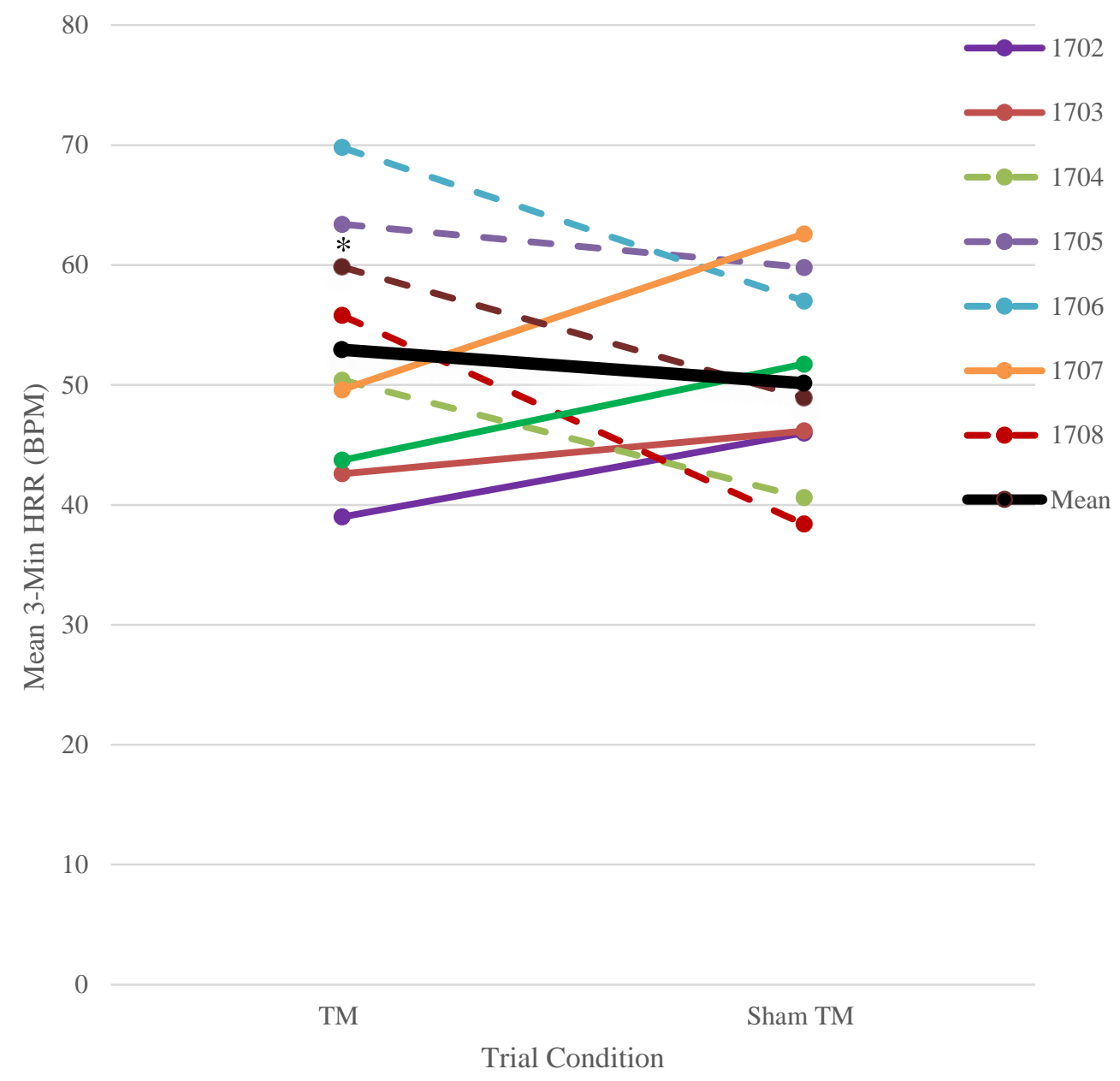

Figure 4.5 Mean 3-Min HRR during TM and Sham TM conditions. *Significant difference between responders and non-responders $(p<0.005)$ in TM condition. - - Responders and - Non-Responders

\section{Stroke Volume}

Mean SV was calculated for each 3-min recovery. Descriptive statistics are presented in Figure 4.6. Repeated measures ANOVA found no significant main effects for trial condition, trial condition*interval ( $p=0.672$ and 0.147 , respectively). Post hoc analysis found that interval one was had a significantly higher mean 3-min recovery SV than intervals three, four, and five ( $p=0.20,0.21$, and 0.24 , respectively). There were no significant differences between responders and non-responders in either condition ( $p=$ 0.204 and 0.527 , respectively); however, Figure 4.6 shows that responders had a higher 
mean 3-min recovery SV than non-responders in the TM condition (111.05 \pm 18.16 vs. $95.8 \pm 8.84)$.

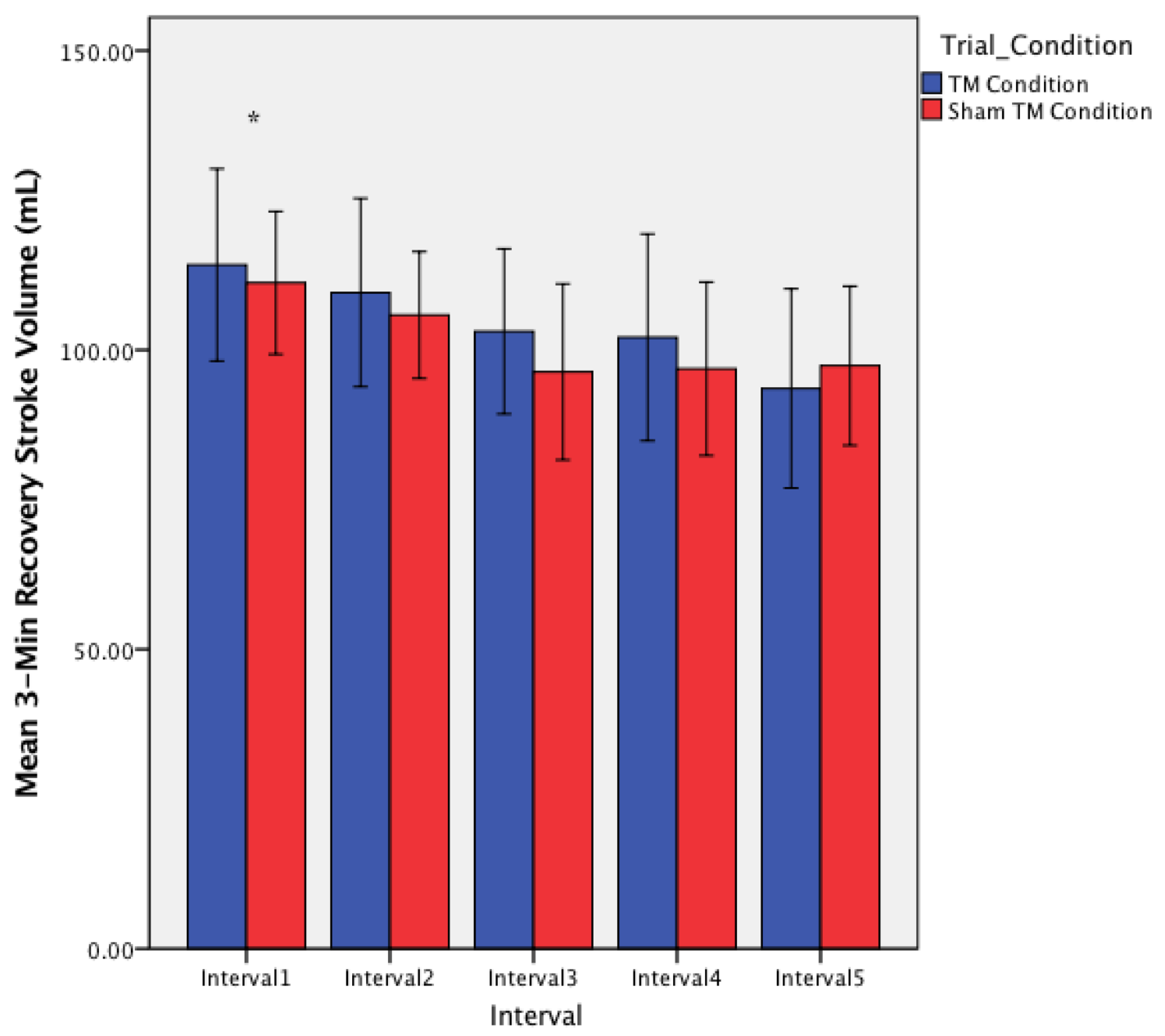

Figure 4.6 Mean ( \pm SD) SV during 3-Min Recovery for each Condition and Interval. *Interval one was significantly greater than intervals three, four, and five $(p=0.20,0.21$, and 0.24 , respectively). 


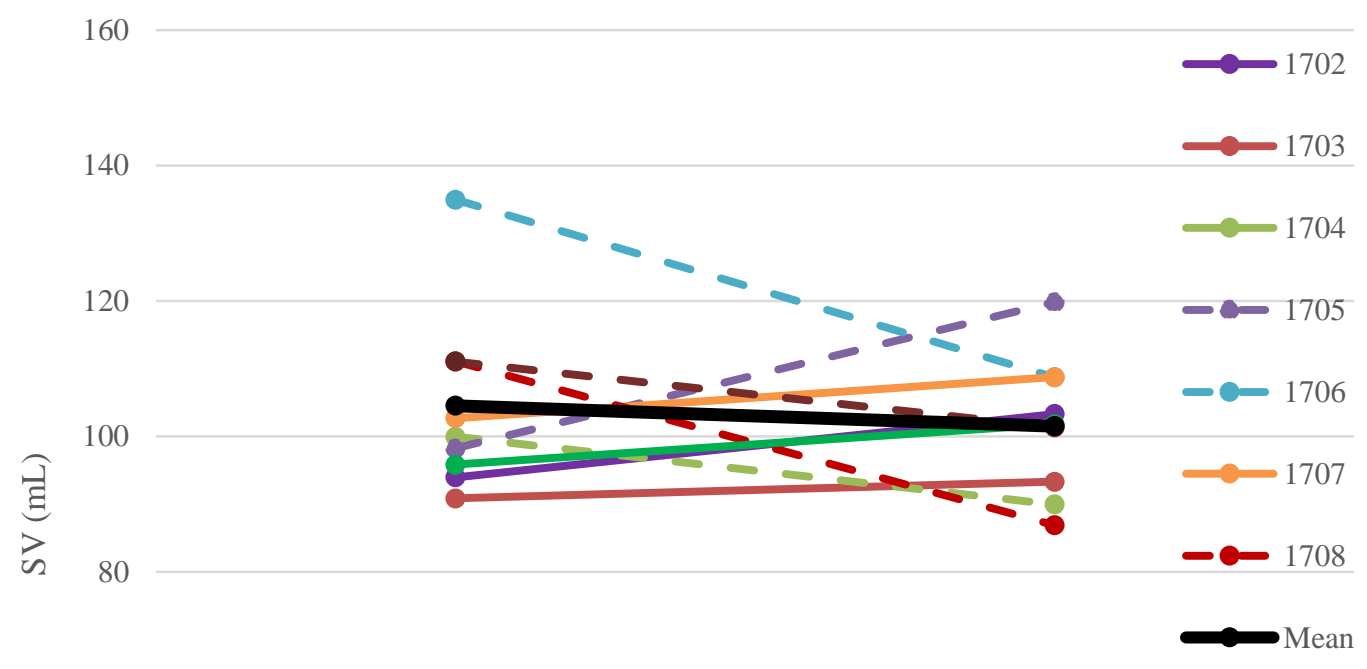

60

40

20

0

$\mathrm{TM}$

Sham TM

Trial Condition

Figure 4.7 Mean 3-min Recovery SV for TM and Sham TM Conditions - - Responders and - Non-Responders

\section{Cardiac Output}

Mean Q was calculated for each 3-min recovery. Descriptive statistics are presented in Figure 4.8. Repeated measures ANOVA found no significant main effects for trial condition, interval, or trial condition*interval $(p=0.775,0.704$, and 0.698 , respectively). 


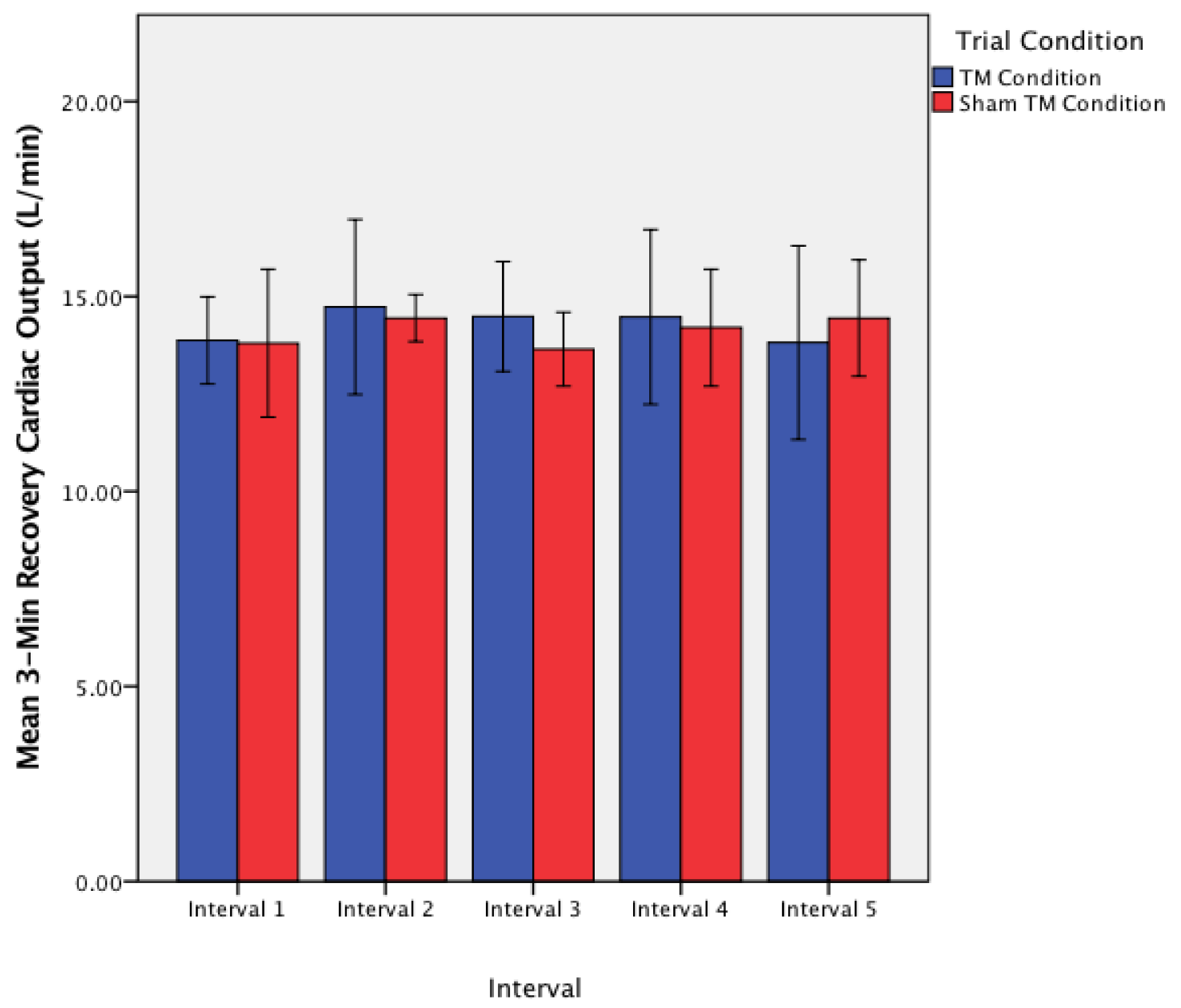

Figure 4.8 Mean $( \pm$ SD) $\dot{Q}$ during 3-Min Recovery for each Condition and Interval.

\section{HRV}

HRV R-R data from the ZB was post-processed in Kubios HRV Premium (Version 3.0.2) (Kuopio, Finland). The very strong threshold-based R-R correction was completed to remove any artifact. HRV data was calculated to determine the change from the first $30 \mathrm{sec}$ to the final $30 \mathrm{sec}$ of each 3-min recovery using the RMSSD (ms). Descriptive statistics are presented in Figure 4.9. A repeated measures ANOVA found no significant main effect for trial condition or trial condition*interval $(p=0.158,0.998$, respectively). There was a significant main effect for interval $(p<0.005)$; however, upon 
post hoc analysis there were no significantly different intervals in either condition $(p>$ $0.05)$.

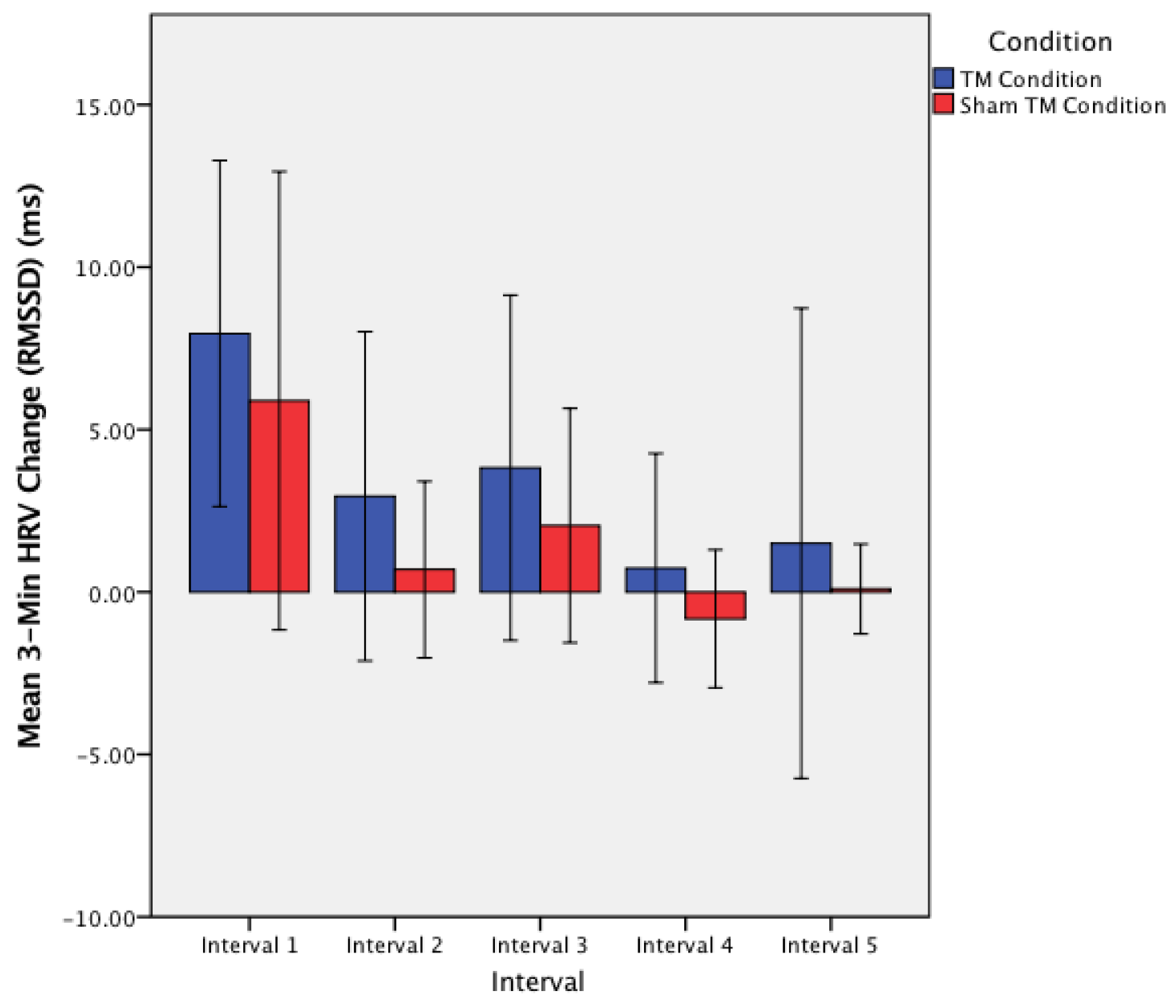

\section{Figure 4.9 Change in Mean ( \pm SD) HRV During 3-Min Recovery Breath Rate Recovery}

Breath Rate Recovery was calculated by subtracting the breath rate following the 1-min row and the breath rate following the 3-min recovery. Descriptive statistics are presented in Table 4.8 and Figure 4.9. A repeated measures ANOVA found a significant main effect for condition ( $p=0.008$ ), while no main effect for interval or trial condition*interval ( $p=0.599$ and 0.267 , respectively). Post Hoc analysis for the simple main effects of condition resulted in TM intervals one, two, and five significantly greater 
breath rate recovery than Sham TM intervals one, two, and five ( $p=0.017,0.019$, and 0.033, respectively). TM Interval three and four were not significantly different in breath rate recovery than Sham TM intervals three and four ( $p=0.310$ and 0.094 , respectively).

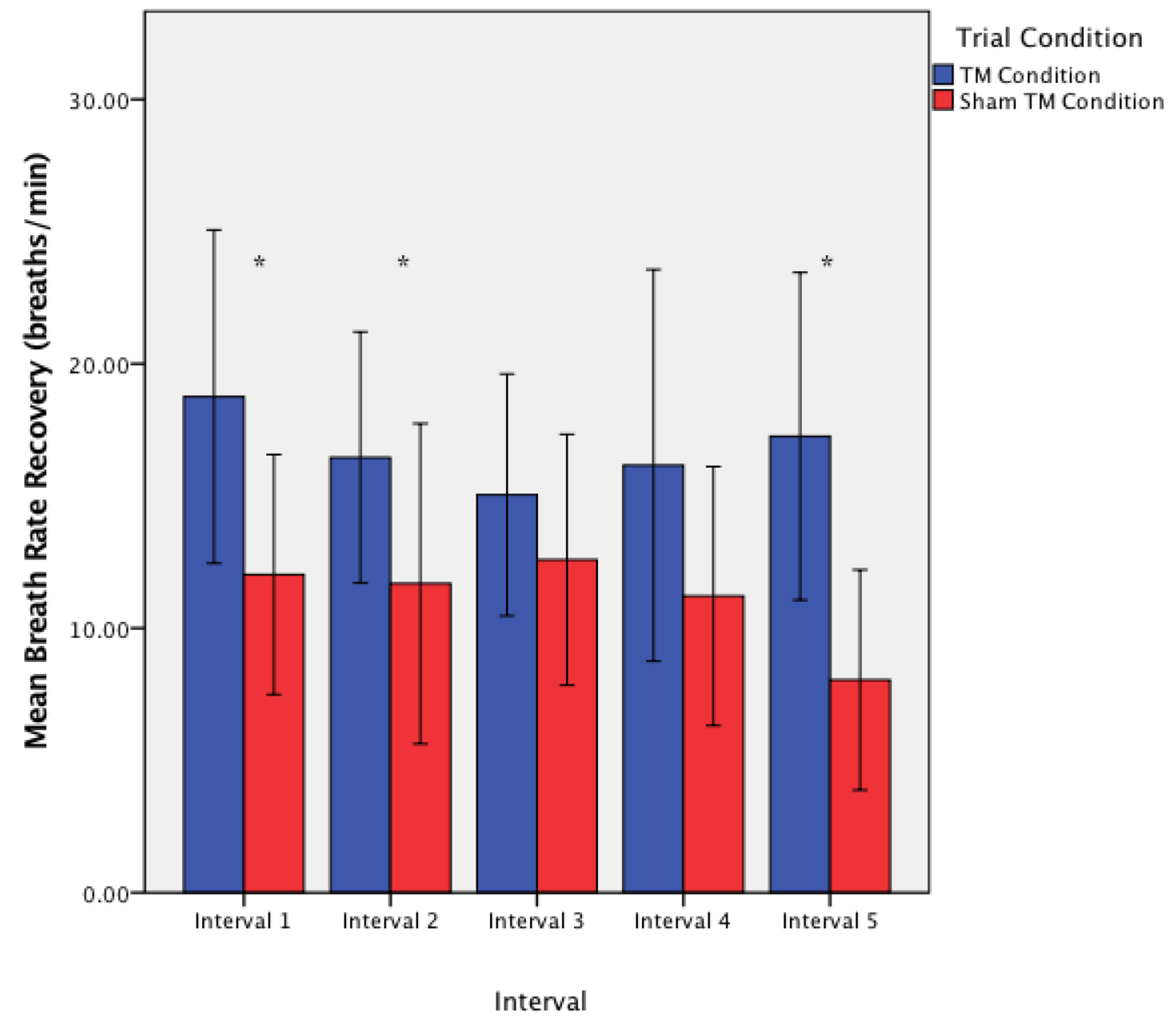

Figure 4.10 Mean ( \pm SD) 3-Min Breath Rate Recovery for Each Condition and Interval. *Significant difference between TM and Sham TM conditions $(p<0.05)$. 


\section{CHAPTER FIVE: DISCUSSION}

The purpose of the present study was to examine the acute effects of the TM on short-term recovery during rowing intervals. Based on anecdotal reports of enhanced HRR and performance when the TM is used during recovery, the author developed four primary hypotheses. $^{22}$

\section{Table 5.1 Hypotheses Following the Results}

\begin{tabular}{|c|c|}
\hline Hypothesis & Accepted/Rejected \\
\hline $\begin{array}{c}\text { HRR, HRV, and performance }(\mathrm{m} / \mathrm{W} \text { ) would be greater } \\
\text { across each repeated rowing interval when the TM is used during } \\
\text { recovery. }\end{array}$ & Rejected \\
\hline $\begin{array}{c}\text { The use of the TM would enhance } \mathbf{S V} \text { and } \mathbf{Q} \text { during the } \\
\text { recovery portion of the interval, and subsequently increase HRR. }\end{array}$ & Rejected \\
\hline $\begin{array}{c}\text { The ventilatory resistance of the TM would slow ventilation } \\
\text { and therefore increase BRR during recovery. }\end{array}$ & Accepted \\
\hline $\begin{array}{c}\text { Blood lactate accumulation over the duration of the interval } \\
\text { training protocol would be less when the TM was used during the } \\
\text { recovery portion of the interval. }\end{array}$ & Rejected \\
\hline
\end{tabular}

Upon further analysis, it was determined that there were four responders and three non-responders of the TM. A TM responder was defined as a participant that had increased HRR in the TM condition. However, it remains unclear what made some participants responders and others non-responders. It is possible that responders utilized diaphragmatic or abdominal breathing patterns, which may have led to increased SV and therefore, decreased HR to maintain an equal Q with non-responders. 


\section{Performance}

This is the first study, to the author's knowledge, that has examined the acute effects of the TM or a respiratory muscle trainer (RMT) used during recovery on performance during anaerobic interval training on a rowing ergometer. The study was a trial to provide a novel use and to expand the TM's practical implications by enhancing short-term recovery, in attempts to enhance short-term recovery and allow participants to continue to perform anaerobic HIIT at higher levels of intensity.

In contrast to the anecdotal reports, there were no differences in performance (m) between the TM and Sham TM conditions. Previous studies on RMT and inspiratory muscle training (IMT) have had mixed results on their efficacy for increasing sport performance; however, it is accepted that RMT can improve performance when the ventilatory demands of the RMT match that of the sport and intensity of the RMT is high enough. ${ }^{45}$ Mechanisms for increased performance that have been examined are reduction in RPE and respiratory muscle fatigue, improved $\dot{\mathrm{VO}}_{2 \max }$, blood lactate clearance, respiratory muscle strength, time trial performance, and repeated sprint ability. ${ }^{45}$

Performance declined across each interval on an average of $1.29 \%$ and $1.52 \%$ for the TM and Sham TM conditions, respectively. This relatively small interval-to-interval decrease in performance indicates that the recovery time was adequate for the present anaerobic interval training protocol to avoid significant acidosis and thus, a greater decline in performance across intervals. ${ }^{19}$ Interestingly, the responders had a slightly less decline in performance across each interval during the TM condition than non-responders $(0.66 \mathrm{vs}$ $2.11 \%$, respectively; $p=0.293$ ). There were no differences between responders and nonresponders in performance decline during the Sham TM condition (1.62 and 1.39\%, 
respectively; $p=0.834)$. Regardless, performance data between the TM and Sham TM conditions were not significantly different and indicates that the use of the TM during recovery did not affect performance. In contrast, Chiappa et al. found that inspiratory resistive loading (IRL) used during a 15-min recovery between two 30-sec Wingates reduced blood lactate accumulation and subsequently improved performance (i.e. peak and mean power, but also increased fatigue index) in the second Wingate. ${ }^{46}$ Chiappa et al. attributed the improved blood lactate clearance to the diaphragm and accessory respiratory muscles as consumers of blood lactate post-exercise. ${ }^{46-48}$ In the present study, there was no significant difference in post-trial blood lactate accumulation between conditions. However, blood lactate concentrations were $7.15 \%$ lower in the TM condition. That being stated, it is not possible to conclude that the TM increased blood lactate clearance, as blood lactate was not measured at the end of each 1-min row and 3min recovery. Regardless, Chiappa et al. were the first to report positive effects of inspiratory resistance when used during recovery between two bouts of high-intensity, anaerobic exercise. Brown et al. found similar results when using IRL on enhancing lactate recovery kinetics. ${ }^{49}$ Participants completed a 6-week IMT program consisting of 30 breaths with the POWERbreathe ${ }^{\circledR}$ IMT at a resistance of $50 \%$ maximal inspiratory pressure, twice daily. Blood lactate recovery following a maximal incremental cycling test was improved when IRL (inspiratory resistance at $15 \mathrm{~cm} \mathrm{H}_{2} \mathrm{O}$ ) was used during a 20min recovery compared to a control group. It is important to note that HRR in the 20-min recovery was not different between IRL and control groups. ${ }^{49}$ Secondly, it is important to note that improved blood lactate kinetics with IRL during recovery were only observed in IMT trained individuals and not in individuals with untrained respiratory muscles. ${ }^{49}$ In 
the present study, three of the four responders reported having used the TM or a RMT prior to participating in the study. This may explain why some participants were responders and others were not. None of the non-responders had used the TM or a RMT prior to the study. Finally, all participants in the present study used the same inspiratory resistance $(3,000 \mathrm{~m})$. It is possible that the relative intensity of the TM may have influenced whether or not the participant was a responder.

In addition to the work of Chiappa et al. there have been studies to examine the acute effects of RMT, but with the use of an IMT warm-up, on performance in rowing and cycling HIIT. ${ }^{50,51}$ Voliantitis et al found that the acute use of the POWERbreathe ${ }^{\circledR}$ IMT (IMT Technologies Ltd, Birmingham, UK) for a respiratory warm-up, in addition to a specific rowing warm-up, increased 6-min all-out rowing performance by $1.2 \%$ and $3.2 \%$ while reducing exertional dyspnea compared to specific or submaximal rowing warm-up, respectively. ${ }^{50}$ In contrast, Ohya et al found no improvement in performance during cycling HIIT following an IMT warm-up. ${ }^{51}$ It is important to note that the later study was on untrained males and may explain the difference in results.

The success of RMT or IMT has shown mixed results when used acutely. It is possible that the chronic training with the TM, a RMT, or an IMT may have potential significant implications. Chronic use of the TM may lead to decreased psychological distress and dyspnea and allow participants to slow their BR and control their breathing mechanics. With three of the four responders having utilized the TM prior to the study, the previous experience may have led to better results. 


\section{HRR and Potential Mechanisms for Enhanced HRR}

HRR was not significantly different between conditions; however, trends showed that the TM condition had slightly improved HRR. These results may have reached statistical significance with a larger sample size. The TM condition produced even more pronounced increases in HRR for responders. Responders had 25\% improved HRR in the TM condition, while non-responders had 14\% improved HRR in the Sham TM condition. When looking at responders only, the HRR results are in agreement with the hypothesis that the TM would increase HRR. This is important to note because the TM may be beneficial for some, while not for others. This should be considered when interpreting the results of the present study. Two primary possible mechanisms were hypothesized for enhanced HRR during the TM condition; however, enhanced SV is a likely justification.

This mechanistic explanation may be due to enhanced venous return through the “respiratory pump.” SV and Q were measured every 30 sec during each 3-min recovery. Mean SV and Q were calculated for each 3-min recovery. While there were no differences in mean SV or $\dot{Q}$ between conditions, responders, while not statistically significant, had a higher mean SV during the TM condition than the non-responders (111.05 \pm 18.16 vs. $95.8 \pm 8.84$ ), while there was no difference in Sham TM condition (101.77 \pm 16.04 vs. $101.51 \pm 10.59)$. Q was also not significantly different between TM and Sham TM conditions, nor between responders and non-responders. Previous literature has shown that the use of an inspiratory resistance device can significantly increase SV and $\dot{Q}$ at rest. ${ }^{24}$ Convertino et al. found that breathing through an inspiratory resistance device increased negative thoracic pressure during inspiration, and thus increased venous return, and thus increased SV and $\dot{Q}$ via a 'vacuum-like' effect in the 
thorax creating a Frank-Starling effect. On the contrary, Miller el al. examined the effects of various breathing patterns (normal, diaphragmatic, and ribcage) and respiratory resistance in a series of studies. ${ }^{31,34}$ Miller et al. found that diaphragmatic and ribcage, but not normal breathing led to increased negative thoracic pressure during inspiration, and thus increased venous return during inspiration, but a positive thoracic pressure during expiration which negated the enhanced venous return during inspiration. ${ }^{31}$ The same response was observed during a follow-up study with a respiratory resistance device. ${ }^{34}$ The respiratory resistance device used in the follow-up study resisted ventilation in both inspiration and expiration. In the present study, the TM only provided inspiratory resistance. This could possibly explain the positive effects observed in the responders and is in agreement with the findings of Convertino et al. ${ }^{24}$

In the present study, breathing technique was not objectively measured; however, BRR was measured. The TM condition led to a significantly higher BRR compared to the Sham TM condition. Thus, ventilatory resistance adequately reduced breath rate, but may or may not have altered the type of breathing (diaphragmatic, abdominal, or accessory muscle). It may be possible that responders, rather than non-responders, altered their breathing technique, which may have led to increased "respiratory pump" activation; however, this was not determined.

The second hypothesized, but less likely, mechanism for enhanced HRR was a shift in the autonomic nervous system from sympathetic dominance to a more parasympathetic tone. HRV was used as a non-invasive measure of autonomic nervous system activity. There were no significant differences in HRV change during each 3-min between TM and Sham TM conditions. There were also no differences between 
responders and non-responders. This supports the hypothesis that the differences in HRR between responders and non-responders was the function of the "respiratory pump" and enhanced SV. In fact, HRV change declined from interval-to-interval indicating increased sympathetic nervous system dominance throughout the trial. This is in agreement with Mourot et al., who found that HIIT led to an increased sympathetic dominance and parasympathetic withdrawal compared to submaximal, continuous exercise. ${ }^{15} \mathrm{HRV}$ was still significantly lower than resting HRV values 20 min post-exercise. Mourot et al. found that it took almost one hour to return to pre-exercise values. ${ }^{15}$ That being said, it is unlikely that the TM condition was able to shift the autonomic nervous system from sympathetic to parasympathetic dominance during any of the 3-min recoveries.

There are limitations to the present study. First, the sample size was relatively small. Large individual variance led to insignificant differences between the TM and Sham TM conditions, as well as across the five intervals. A larger sample size could have accounted for and reduced the impact of the individual differences observed within each trial condition. That being stated, the participants that were recruited were well-trained. For context, the mean "Jackie" time was 7:43.9 min, while national competitors' mean time was 5:50.4 min. This puts the participants' mean time within two min. A novice-torecreationally trained individual's time for this workout would be $>14 \mathrm{~min}$. A second limitation to the study was that three of the seven participants reported having used the $\mathrm{TM}$ or a respiratory muscle trainer prior to participating in the study; however, neither the extent nor the context of the use of a TM or respiratory muscle trainer was reported. This limitation is important to note, as the novelty and inexperience of using a respiratory muscle trainer may have led to decreased desired physiological responses to the TM 
during short-term recovery or possibly have led to increased psychological distress and thus, pacing or decreased performance. In the present study, the familiarization consisted of one interval with the TM and one without the TM. Future studies should implement a full familiarization trial to try to delimit any confounding effects of TM or RMT experience. A third limitation was rowing technique. While the inclusion requirement of a sub-9 min "Jackie" time was used to attempt to ensure a proficient rower, the technique used by individual participants was not controlled. This may have led to decreased performance. A final limitation was current training status of the participants. One participant reported a cessation of training for three weeks between visits two and three, while another participant reported being ill between trials and current training was blunted. These reports may have led to decreased performance between trials.

\section{Future Research}

Future research should address the RMT and TM familiarization limitations to the present study. It would be warranted to exclude potential participants who had used a RMT or TM prior to the study or to include a more extensive familiarization in attempt to delimit potential confounding from experience. Future research should investigate longitudinal training with the TM or RMT used during short-term recovery and examine the effects on performance, HRR, and blood lactate clearance. The present study observed responders and non-responders. Future research should investigate possible mechanisms for a response vs. no response to the TM. It may be beneficial to utilize different or various RMTs and use a relative, rather than fixed resistance for each participant. Other research may focus on measuring or controlling breathing techniques through electromyography. 


\section{Conclusions}

Contrary to anecdotal reports of the success of the TM used during short-term recovery between intervals, the present study showed no difference in performance between Sham TM and TM conditions. Interestingly, there were responders and nonresponders to the TM for HRR. It was shown that responders had an increased SV during recovery, but $\dot{Q}$ was equivalent between responders and non-responders. HRV declined from interval to interval and showed that sympathetic nervous system dominance increased across time. Neither ventilatory resistance nor potential changes in breathing mechanics could reactivate the parasympathetic nervous system during short-term recovery. That being stated, further investigation is warranted into whether or not there is potential performance or training augmentation from the use of the TM during short-term recovery in anaerobic HIIT. This study did, however, produce some novel physiological responses to ventilatory resistance. The dichotomy of responses to the TM reveals avenues for future research. 


\section{REFERENCES}

1. Jones AM, Carter $\mathrm{H}$. The effect of endurance training on parameters of aerobic fitness. Sports Med. 2000;29(6):373-386.

2. Billat LV. Interval training for performance: a scientific and empirical practice. Special recommendations for middle- and long-distance running. Part I: aerobic interval training. Sports Med. 2001;31(1):13-31.

3. Gastin PB. Energy system interaction and relative contribution during maximal exercise. Sports Med. 2001;31(10):725-741.

4. Billat LV. Interval training for performance: a scientific and empirical practice. Special recommendations for middle- and long-distance running. Part II: anaerobic interval training. Sports Med. 2001;31(2):75-90.

5. Svedahl K, MacIntosh BR. Anaerobic threshold: the concept and methods of measurement. Can J Appl Physiol. 2003;28(2):299-323.

6. Faiss R, Girard O, Millet GP. Advancing hypoxic training in team sports: from intermittent hypoxic training to repeated sprint training in hypoxia. Br J Sports Med. 2013;47 Suppl 1:i45-50.

7. Burgomaster KA, Hughes SC, Heigenhauser GJ, Bradwell SN, Gibala MJ. Six sessions of sprint interval training increases muscle oxidative potential and cycle endurance capacity in humans. J Appl Physiol (1985). 2005;98(6):1985-1990. 
8. Daussin FN, Ponsot E, Dufour SP, et al. Improvement of VO2max by cardiac output and oxygen extraction adaptation during intermittent versus continuous endurance training. Eur J Appl Physiol. 2007;101(3):377-383.

9. Pierpont GL, Stolpman DR, Gornick CC. Heart rate recovery post-exercise as an index of parasympathetic activity. J Auton Nerv Syst. 2000;80(3):169-174.

10. Buchheit M, Laursen PB. High-intensity interval training, solutions to the programming puzzle: Part I: cardiopulmonary emphasis. Sports Med. $2013 ; 43(5): 313-338$.

11. Driller MW, Fell JW, Gregory JR, Shing CM, Williams AD. The effects of highintensity interval training in well-trained rowers. Int J Sports Physiol Perform. 2009;4(1):110-121.

12. Buchheit M, Laursen PB, Ahmaidi S. Parasympathetic reactivation after repeated sprint exercise. Am J Physiol Heart Circ Physiol. 2007;293(1):H133-141.

13. Buchheit M, Gindre C. Cardiac parasympathetic regulation: respective associations with cardiorespiratory fitness and training load. Am J Physiol Heart Circ Physiol. 2006;291(1):H451-458.

14. Goldberger JJ, Le FK, Lahiri M, Kannankeril PJ, Ng J, Kadish AH. Assessment of parasympathetic reactivation after exercise. Am J Physiol Heart Circ Physiol. 2006;290(6):H2446-2452.

15. Mourot L, Bouhaddi M, Tordi N, Rouillon JD, Regnard J. Short- and long-term effects of a single bout of exercise on heart rate variability: comparison between constant and interval training exercises. Eur J Appl Physiol. 2004;92(4-5):508517. 
16. Bishop PA, Jones E, Woods AK. Recovery from training: a brief review: brief review. J Strength Cond Res. 2008;22(3):1015-1024.

17. Cottrell GT, Coast JR, Herb RA. Effect of recovery interval on multiple-bout sprint cycling performance after acute creatine supplementation. $J$ Strength Cond Res. 2002;16(1):109-116.

18. Engles H, Kolokouri I, Cieslak T, Wirth J. Effects of ginseng supplementation on supra-maximal exercise performance and short-term recovery. Journal of Strength and Conditioning Research. 2001;15:290-295.

19. Glaister M. Multiple sprint work : physiological responses, mechanisms of fatigue and the influence of aerobic fitness. Sports Med. 2005;35(9):757-777.

20. Seiler S, Hetlelid KJ. The impact of rest duration on work intensity and RPE during interval training. Med Sci Sports Exerc. 2005;37(9):1601-1607.

21. Jerath R, Edry JW, Barnes VA, Jerath V. Physiology of long pranayamic breathing: neural respiratory elements may provide a mechanism that explains how slow deep breathing shifts the autonomic nervous system. Med Hypotheses. 2006;67(3):566-571.

22. Mackenzie B. Interval breathing recovery protocol. 2015. Accessed April, 3, 2016.

23. Porcari JP, Probst L, Forrester K, et al. Effect of Wearing the Elevation Training Mask on Aerobic Capacity, Lung Function, and Hematological Variables. J Sports Sci Med. 2016;15(2):379-386. 
24. Convertino VA, Ratliff DA, Ryan KL, et al. Hemodynamics associated with breathing through an inspiratory impedance threshold device in human volunteers. Crit Care Med. 2004;32(9 Suppl):S381-386.

25. Janse de Jonge XA. Effects of the menstrual cycle on exercise performance. Sports Med. 2003;33(11):833-851.

26. Baechle T, Earle R. Essentials of Strength Training and Conditioning. 3 ed. Champaign, IL: Human Kinetics; 2008.

27. Seiler S, Sjursen JE. Effect of work duration on physiological and rating scale of perceived exertion responses during self-paced interval training. Scand J Med Sci Sports. 2004;14(5):318-325.

28. Fox EL, Bartels RL, Billings CE, O'Brien R, Bason R, Mathews DK. Frequency and duration of interval training programs and changes in aerobic power. $J$ Appl Physiol. 1975;38(3):481-484.

29. Stevens AW, Olver TT, Lemon PW. Incorporating sprint training with endurance training improves anaerobic capacity and 2,000-m Erg performance in trained oarsmen. J Strength Cond Res. 2015;29(1):22-28.

30. Pelka M, Kölling S, Ferrauti A, Meyer T, Pfeiffer M, Kellmann M. Acute effects of psychological relaxation techniques between two physical tasks. J Sports Sci. 2016:1-8.

31. Miller JD, Pegelow DF, Jacques AJ, Dempsey JA. Skeletal muscle pump versus respiratory muscle pump: modulation of venous return from the locomotor limb in humans. J Physiol. 2005;563(Pt 3):925-943. 
32. Uva B, Aliverti A, Bovio D, Kayser B. The "Abdominal Circulatory Pump": An Auxiliary Heart during Exercise? Front Physiol. 2015;6:411.

33. Byeon $\mathrm{K}$, Choi JO, Yang JH, et al. The response of the vena cava to abdominal breathing. J Altern Complement Med. 2012;18(2):153-157.

34. Miller JD, Pegelow DF, Jacques AJ, Dempsey JA. Effects of augmented respiratory muscle pressure production on locomotor limb venous return during calf contraction exercise. J Appl Physiol (1985). 2005;99(5):1802-1815.

35. Patwardhan AR, Vallurupalli S, Evans JM, Bruce EN, Knapp CF. Override of spontaneous respiratory pattern generator reduces cardiovascular parasympathetic influence. J Appl Physiol (1985). 1995;79(3):1048-1054.

36. Leaderboard. 2013. Accessed April, 11, 2016.

37. PAR-Q \& You. 2002. Accessed April, 18, 2016.

38. Training Mask. 2016. Accessed April, 17, 2016.

39. Johnstone JA, Ford PA, Hughes G, Watson T, Garrett AT. Bioharness( ${ }^{\text {TM }}$ ) multivariable monitoring device: part. I: validity. J Sports Sci Med. 2012;11(3):400-408.

40. Johnstone JA, Ford PA, Hughes G, Watson T, Garrett AT. Bioharness( ${ }^{\text {TM }}$ ) Multivariable Monitoring Device: Part. II: Reliability. J Sports Sci Med. 2012;11(3):409-417.

41. Teboul JL, Saugel B, Cecconi M, et al. Less invasive hemodynamic monitoring in critically ill patients. Intensive Care Med. 2016;42(9):1350-1359. 
42. Keren H, Burkhoff D, Squara P. Evaluation of a noninvasive continuous cardiac output monitoring system based on thoracic bioreactance. Am J Physiol Heart Circ Physiol. 2007;293(1):H583-589.

43. Hart S, Drevets K, Alford M, Salacinski A, Hunt BE. A method-comparison study regarding the validity and reliability of the Lactate Plus analyzer. BMJ Open. $2013 ; 3(2)$.

44. Moran P, Prichard JG, Ansley L, Howatson G. The influence of blood lactate sample site on exercise prescription. J Strength Cond Res. 2012;26(2):563-567.

45. HajGhanbari B, Yamabayashi C, Buna TR, et al. Effects of respiratory muscle training on performance in athletes: a systematic review with meta-analyses. $J$ Strength Cond Res. 2013;27(6):1643-1663.

46. Chiappa GR, Ribeiro JP, Alves CN, et al. Inspiratory resistive loading after all-out exercise improves subsequent performance. Eur J Appl Physiol. 2009;106(2):297303.

47. Chiappa GR, Roseguini BT, Neder JA, Ribeiro JP. Inspiratory loading and lactate clearance after exercise. Med Sci Sports Exerc. 2013;45(1):212-213.

48. Chiappa GR, Roseguini BT, Alves CN, Ferlin EL, Neder JA, Ribeiro JP. Blood lactate during recovery from intense exercise: impact of inspiratory loading. Med Sci Sports Exerc. 2008;40(1):111-116.

49. Brown PI, Sharpe GR, Johnson MA. Loading of trained inspiratory muscles speeds lactate recovery kinetics. Med Sci Sports Exerc. 2010;42(6):1103-1112. 
50. Volianitis S, McConnell AK, Koutedakis Y, Jones DA. Specific respiratory warm-up improves rowing performance and exertional dyspnea. Med Sci Sports Exerc. 2001;33(7):1189-1193.

51. Ohya T, Hagiwara M, Suzuki Y. Inspiratory muscle warm-up has no impact on performance or locomotor muscle oxygenation during high-intensity intermittent sprint cycling exercise. Springerplus. 2015;4:556. 
APPENDIX A

Informed Consent Form 


\title{
B
}

BOISE STATE UNIVERSITY

\section{INFORMED CONSENT}

\author{
Study Title: Acute Effects of the Training Mask on Short-Term Recovery \\ During Rowing Intervals \\ Principal Investigator: Hayden \\ Hess \\ Co-Investigator: Dr. Shawn \\ Simonson
}

Sponsor: N/A

This consent form will give you the information you will need to understand why this research study is being done and why you are being invited to participate. It will also describe what you will need to do to participate as well as any known risks, inconveniences or discomforts that you may have while participating. We encourage you to ask questions at any time. If you decide to participate, you will be asked to sign this form and it will be a record of your agreement to participate. You will be given a copy of this form to keep.

\section{$>$ PURPOSE AND BACKGROUND}

The purpose of the present study is to examine the effects of the Training Mask on heart rate, heart rate variability (variability in time between subsequent heart beats), heart rate recovery, cardiac output, and performance across multiple maximal intensity intervals on the rowing ergometer, when the Training Mask is used during the recovery (rest) portion of interval training. To be in this study, you must be a male between 18 and 45 years of age, in good physical health (no diagnosed cardiovascular, pulmonary, metabolic, musculoskeletal, or chronic disease), and have completed the CrossFit workout "Jackie" (1,000m row, 5045 -pound Thrusters, and 30 Pull-ups) in 14 min or less.

\section{PROCEDURES}

If you agree to be in this study, you will participate in the following:

- Visit 1: Orientation and Familiarization - approximately one hour.

- Visit 2 and 3: Exercise Trials - approximately one hour each.

You will be asked to come to the Human Performance Laboratory in the Norco Building on three occasions. Prior to the first interval training trial (Visit 2) you will be required to bring a 24-hour food and hydration log. Prior to the second interval training trial (Visit 3), you will be asked to have repeated the same 24-hour food and hydration intake. You will also be required to have had 24-hours rest (no exercise) prior to Visits 2 and 3. You will be asked to not consume caffeine or other ergogenic (performance enhancing) supplements prior to Visits 2 and 3 . You will be asked not to consume any food 3 hours prior to Visits 2 and 3, but may eat 1 apple, 1 
hour prior to Visits 2 and 3 if you are inclined.

Visit 1 - Paperwork, Orientation, and Familiarization (1 Hour)

During the first visit, you will complete all paperwork (informed consent, modified Physical Activity Readiness Questionnaire, training history and demographic questions) and be debriefed on the study and its protocol. Following the briefing, you will be familiarized with the interval training protocol and the Training Mask. You will complete one interval with the Training Mask (with resistance) and one interval with the Training Mask (no resistance).

\section{Visits 2 and 3 - Interval Training Trials (1 Hour each visit)}

You will complete one of two interval training protocols on the rowing ergometer. You will either complete the interval training protocol with the Training Mask (with resistance) or with the Training Mask (no resistance). You will complete each protocol, in random order, on separate Visits (2 and 3).

\section{$>$ RISKS}

The potential risks that may occur with participating in this study include those related with exercise. These include muscle/joint soreness, lightheadedness, nausea, and in rare instances, fainting and heart attack. However, the possibility of serious events happening in fit people who have no previous history of heart disease is extremely low. The Human Performance Laboratory has an emergency action plan and all research personnel are CPR/AED certified.

\section{BENEFITS}

There will be no direct benefit to you from participating in this study. However, the information that you provide may help develop improved training and recovery protocols as well as increase understanding of exercise and recovery regulation.

\section{$>$ EXTENT OF CONFIDENTIALITY}

Reasonable efforts will be made to keep the personal information in your research record private and confidential. Any identifiable information obtained in connection with this study will remain confidential and will be disclosed only with your permission or as required by law. The members of the research team and the Boise State University Office of Research Compliance (ORC) may access the data. The ORC monitors research studies to protect the rights and welfare of research participants.

Your name will not be used in any written reports or publications which result from this research. Data will be kept for three years (per federal regulations) after the study is complete and then destroyed. 
For this research project, the researchers are requesting demographic information. Due to the make-up of Idaho's population, the combined answers to these questions may make an individual person identifiable. The researchers will make every effort to protect your confidentiality. However, if you are uncomfortable answering any of these questions, you may leave them blank.

\section{$>$ PAYMENT}

You will not be paid for your participation in this study. However, following full completion of the study, a complimentary testing service (body composition, resting metabolic rate, maximal exercise test) will be offered and provided by the principal investigator.

\section{PARTICIPATION IS VOLUNTARY}

You are free to make a decision to participate in this study, and if you should choose to participate, you may withdraw from the study at any time without penalty. If you withdraw from the study, your data will be destroyed.

\section{$>$ QUESTIONS}

If you have any questions or concerns about your participation in this study, you may contact the Principal Investigator, Hayden Hess: 208-426-5518 or haydenhess@u.boisestate.edu. You may also contact my faculty adviser, Dr. Shawn Simonson: shawnsimonson@boisestate.edu.

If you have questions about your rights as a research participant, you may contact the Boise State University Institutional Review Board, which is concerned with the protection of volunteers in research projects. You may reach the board office between 8:00 AM and 5:00 PM, Monday through Friday, by calling (208) 426-5401 or by writing: Institutional Review Board, Office of Research Compliance, Boise State University, 1910 University Dr., Boise, ID 83725-1138.

\section{DOCUMENTATION OF CONSENT}

I have read this form and decided that I will participate in the project described above. Its general purposes, the particulars of involvement and possible risks have been explained to my satisfaction. I understand I can withdraw at any time.

\begin{tabular}{lll}
\hline $\begin{array}{l}\text { Printed Name of Study } \\
\text { Participant }\end{array}$ & $\begin{array}{l}\text { Signature of Study } \\
\text { Participant }\end{array}$ & Date
\end{tabular}




\section{APPENDIX B \\ Health and Training History Questionnaire and Modified Physical Activity Readiness Questionnaire}




\section{Health/Training History Questionnaire and Modified PAR-Q}

Name:

Age:

Date of Birth:

Gender:

Ethnicity:

Telephone \#:

E-mail Address:

Person to contact in case of an emergency:

Phone \#

Relationship:

\section{Physical Activity Readiness Questionnaire (PAR-Q)}

Please read the questions carefully and answer each honestly:

YES NO

1. Has your doctor ever said that you have a heart condition and that you should only do physical activity recommended by a doctor?

2. Do you feel pain in your chest when you do physical activity?

3. In the past month, have you had chest pain when you were not doing physical activity?

4. Do you lose your balance because of dizziness or do you ever lose consciousness?

5. Do you have a bone or joint problem that could be made worse by a change in your physical activity?

6. Is your doctor currently prescribing drugs (for example, water pills) for your blood pressure or heart condition? 
7. Do you know of any other reason why you should not do physical

activity?

Adapted from PAR-Q \& You (2002).

Have you sustained any orthopedic injuries in the past 6 months? Yes No

If yes, please describe:

Do you have asthma? Yes No

If yes, is it Exercise-Induced? Yes No

\section{Training History}

Do you currently participate in CrossFit? Yes No

How many days per week?

How much time per session?

How long have you been participating in CrossFit?

What is your "Jackie" time?

What is your "Fran" time?

What is your Clean \& Jerk (in pounds)?

What is your Snatch (in pounds)?

If known, what is your $2000 \mathrm{~m}$ row time?

Have you ever participated in a CrossFit competition? Yes_ No

If yes, what competition(s)? 
APPENDIX C

Figure of the Training Mask 2.0 


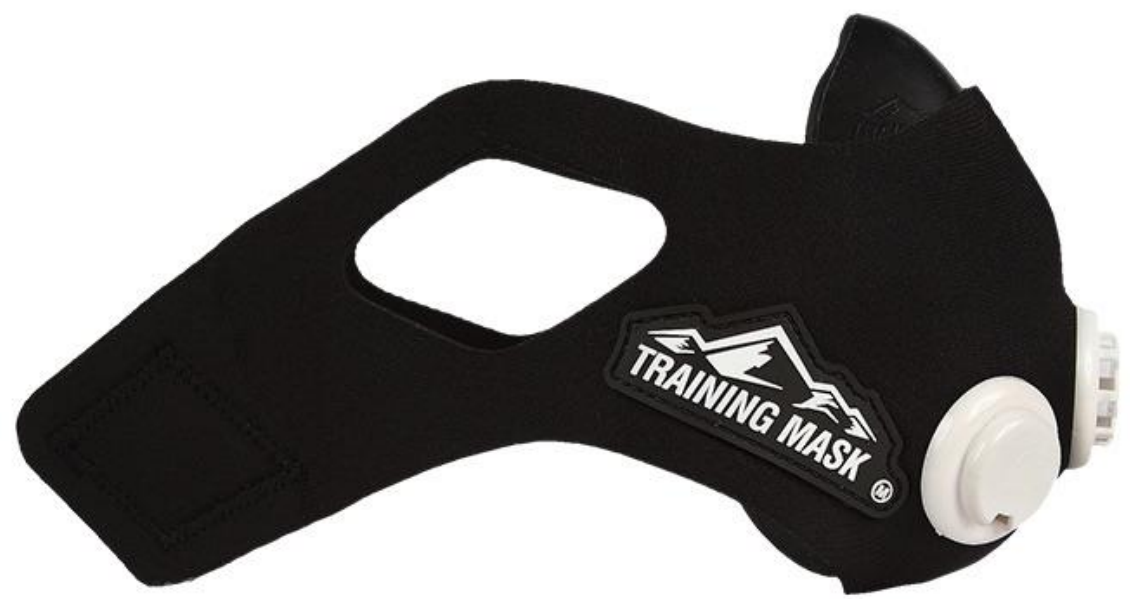

Training Mask 2.0. From Training Mask, 2016. 
APPENDIX D

Participant Compliance Form 


\section{Participant Compliance Form}

$\mathrm{I}$, have to the best of my abilities followed the

instructions to:

- Complete a 24-hour food and hydration log

- Complete a 24-hour rest from physical activity outside of activities of daily living

- Not consumed alcohol in 12 hours

- Not consumed any supplements or ergogenic aids in three hours

- Not consumed any meal in three hours, BUT consumed one apple/banana one hour prior (circle if apply)

Visit 2: Print: Sign:

Date:

Visit 3: Print: Sign:

Date: 
APPENDIX E

Institutional Review Board Protocol 
This research was conducted under approval from the Institutional Review Board at Boise State University, protocol \#103-MED16-008. 